\title{
Chapter 21 \\ Multi-scale Modeling of Partially Stabilized Zirconia with Applications to TRIP-Matrix Composites
}

\author{
Mohan Kumar Rajendran, Michael Budnitzki and Meinhard Kuna
}

\begin{abstract}
The understanding of how the microstructure influences the mechanical response is an essential pre-requisite for materials tailored to match specific requirements. The aim of this chapter is to further this understanding in the context of Mg-PSZ-TRIP-steel composites on three different scales using a set of methods ranging from phase-field simulations over micromechanics to continuum constitutive modeling. On the microscale, using a Ginzburg-Landau type phase-field model the effects of cooling- and stress-induced martensitic phase transformation in $\mathrm{MgO}-\mathrm{PSZ}$ is clearly distinguished. Additionally with this method the role of energy barrier in variant selection and the effect of residual stress contributing to the stability of the tetragonal phase are also investigated. On the mesomechanical scale, an analytical 2D model for the martensitic phase transformation and self-accommodation of inclusions within linear elastic materials has been successfully developed. The influences of particle size and geometry, chemical driving force, temperature and surface energy on the $t \rightarrow m$ transformation are investigated in a thermostatic approach. On the continuum scale, a continuum material model for transformation plasticity in partially stabilized zirconia ceramics has been developed. Nonlinear hardening behavior, hysteresis and monoclinic phase fraction during a temperature cycle are analyzed. Finally, The mechanical properties of a TRIP steel matrix reinforced by $\mathrm{ZrO}_{2}$ particles are analyzed on representative volume elements. Here the mechanical properties of the composite as function of volume fraction of both constituents and the strength of the interface are studied.
\end{abstract}

\footnotetext{
M. K. Rajendran $(\varangle) \cdot$ M. Budnitzki · M. Kuna Institute of Mechanics and Fluid Dynamics, Technische Universität Bergakademie Freiberg, Lampadiusstrasse 4, 09599 Freiberg, Germany e-mail: mohan-kumar.rajendran@imfd.tu-freiberg.de 


\subsection{Introduction}

\subsubsection{Aims and Scopes of the Present Work}

One central aim of the Collaborative Research Center SFB799 "TRIP-matrix composites" was the development of a particle reinforced composite, composed of a TRIP-steel metallic matrix and ceramic particles of partially stabilized zirconia PSZ. The underlying idea was to exploit the phase transformation capability of both constituents in order to enhance and to optimize the mechanical properties by making such a composite.

In particular, the combination of the strain-induced phase transformation in the TRIP steel and the stress-induced transformation in PSZ offers the opportunity to compensate local stress concentrations at considerably high plastic deformations. This reinforcing effect has been verified by several experiments with these metalmatrix-composites MMC under monotonous [1] loading.

In complementation to the fabrication and characterization of these MMC, a thorough theoretical-numerical modeling of the composite material was necessary to understand and to simulate the phase transformation and the deformation behavior of both constituents.

This work was devoted to the simulation of the phase transformation processes in the PSZ ceramics and the MMC, whereas in another work the behavior of the TRIP-steel was investigated. The aim was to provide proper constitutive equations for stress and temperature controlled tetragonal-to-monoclinic phase transformation of PSZ ceramics, based on physical assumptions accounting for the responsible micromechanical mechanisms. The problem has been approached at various length scales, see Fig. 21.1:

- At the microscale level it was needed to simulate the actual kinetics of the transformation process inside of single tetragonal phase particles in PSZ. This task could be best accomplished by using the phase-field method.

- To study the transformation conditions of an ensemble of tetragonal lentils in polycrystalline PSZ ceramics, a semi-analytical thermostatic approach was applied at the mesoscale.

- In order to enable quantitative strength analysis of structures made of PSZ and MMC, a phenomenological constitutive law at the macroscale was further developed and implemented in a FEM-environment.

- To support the development of tailored particle MMC TRIP-matrix composites, representative volume elements on the composite level were simulated, which allow to predict the mechanical properties of the composite as function of volume fraction of both constituents and the strength of the interface. 

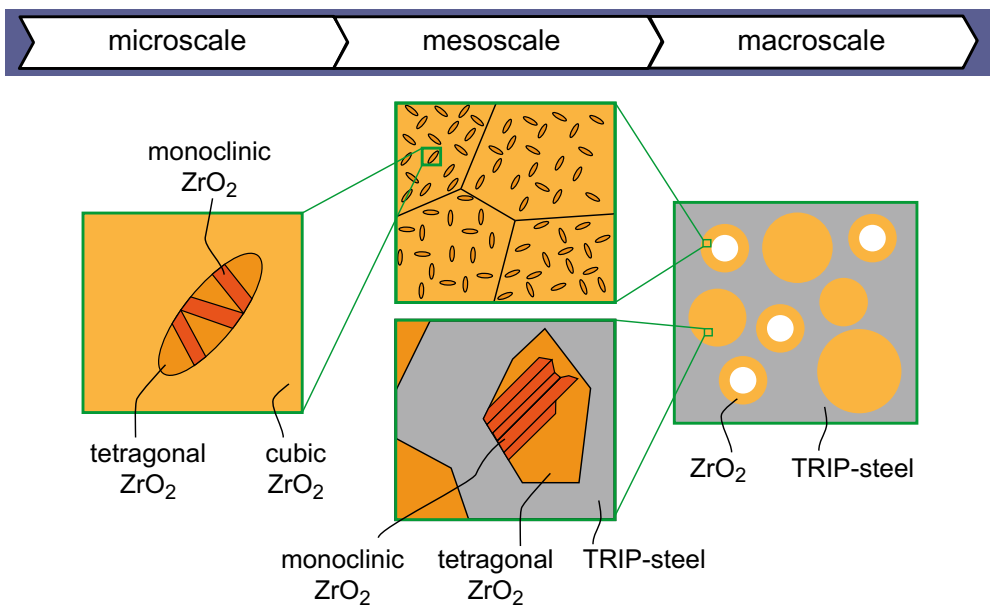

Fig. 21.1 Different scales of modeling partially stabilized zirconia

\subsubsection{Introduction to Partially Stabilized Zirconia}

Partially stabilized zirconia (PSZ) is widely used because of its enhanced fracture toughness and nonlinear stress-strain behavior. These favorable mechanical properties of PSZ result from a solid state phase transformation at regions of high stress concentration (e.g. crack tips). This effect, known as transformation toughening, was first reported by Garvie et al. [2] and was extensively investigated by [3-5]. Generally, some conditions have to be fulfilled for transformation toughening. The existence of a metastable phase in the material is required, which can be achieved either by microstructural parameters such as grain size or by changing the chemical composition. The martensitic (instantaneous) transformation from the metastable parent phase to the stable resultant phase has to be stress-induced.

The PSZ ceramic material under consideration is stabilized by $\mathrm{MgO}$, resulting in finely dispersed lenticular precipitates of tetragonal ( $t$-phase) embedded coherently in the grains of a polycrystalline cubic matrix material (see Fig. 21.2). These precipitates can transform into the monoclinic ( $m$-phase) [7] triggered either by temperature or stress, resulting in the formation of multiple, partially self-accommodating variants. The $t \rightarrow m$ phase transformation, if unconstrained, is accompanied by a volume dilatation of about $4 \%$ and a shear strain of about $8 \%$. The increase in volume induces (residual) compressive stresses in the cubic ( $c$-phase) matrix leading to a shielding effect at stress concentrations, which contributes to the toughness of the material. 
Fig. 21.2 Typical microstructure of a TRIP-PSZ composite produced in the CRC 799 by spark plasma sintering. The image shows a PSZ particle surrounded by TRIP steel together with a zoom into the particle showing the typical PSZ microstructure (reprinted from [6])

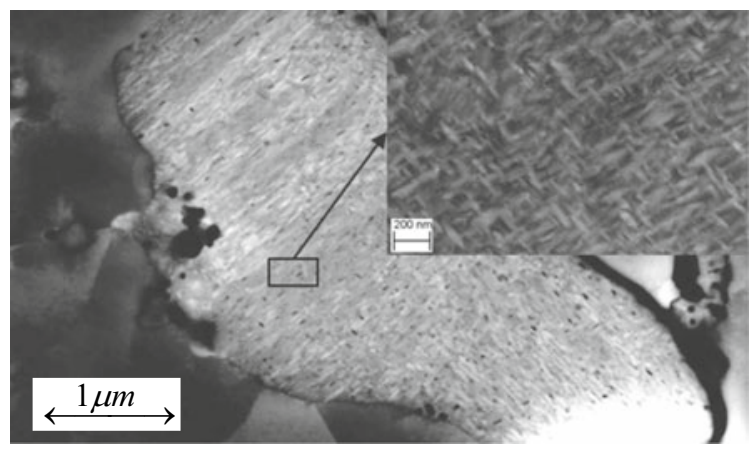

\subsection{Micromechanical Phase-Field Approach}

Phase-field is considered to be a powerful mathematical computational tool in simulations involving interface kinetics. In the past decades PF approach has been successfully established in various fields for materials science understanding such as: solidification, solid-state phase transformation, precipitate evolution and coarsening kinetics, grain growth, martensitic phase transformation (MPT) and also in damage and crack growth phenomena.

For past few decades there has been active research towards the direction of modelling partially stabilized zirconia (PSZ) materials. Wang et al. [8] was one of the early study on PSZ for $c \rightarrow t$ phase transformation involving Ginzburg-Landau (GL) phenomenological theory based PF model. Later in [9] the authors simulated alternating band structure formed by self-organized orientation variants of $t$-phase particles. In [10] the first three-dimensional model for generic $c \rightarrow t$ MPT was presented. Mamivand et al. [11] reported the first work on anisotropic and inhomogeneous PF modeling for $t \rightarrow m$ phase transformation in zirconia ceramics. The work discussed the simulation results based on different initial and boundary conditions in comparison to experimental observations. Further the authors [12] incorporated the effect of stress and temperature to capture the forward $t \rightarrow m$ and reverse $m \rightarrow t$ transformation to model pseudo-elastic behavior in polycrystalline zirconia.

A comprehensive work on non-conserved type GL-based phase-field models for generic martensitic phase transformation was developed in a series of three papers from Levitas et al. [13-15]. Levitas et al. used a $2-3-4$ or higher order polynomial for approximating the Gibbs energy and effective strain transition from austenite to any martensitic variant. This work principally relies on the phenomenological GL phase-field model developed by Levitas et al. with $2-4-6$ type Landau potential. 


\subsubsection{Phase-Field Method}

The temporal and spatial evolution of non-conserved phase-field variables is described by the phenomenological Ginzburg-Landau kinetic equation

$$
\frac{\partial \phi}{\partial t}=-L\left(-\beta \nabla^{2} \phi+\frac{\partial \psi_{\mathrm{gl}}}{\partial \phi}\right) .
$$

Here $L$ is the positive kinetic coefficient, and $\beta$ is a positive gradient energy coefficient. The interface energy contribution provided by Levitas and Preston [13] was used.

Here the total enthalpy $\psi_{\mathrm{gl}}$ is described by additive contributions from an elastic $\psi_{\mathrm{el}}$ and a stress-independent chemical part of free enthalpy $\psi_{\mathrm{ch}}$. The individual contributions are based on the work of Levitas and Preston [13, 14].

$$
\psi_{\mathrm{gl}}=\psi_{\mathrm{el}}\left(\boldsymbol{\epsilon}, \boldsymbol{\epsilon}_{\mathrm{tr}}, \phi\right)+\psi_{\mathrm{ch}}(\theta, \phi)
$$

The order parameter considered here ranges from $-1 \leqslant \phi \leqslant 1$, where $\phi= \pm 1$ are the two possible product variants of $m$-phase and $\phi=0$ represents the parent $t$-phase in two dimensions, see Fig. 21.3b. The values \pm 1 correspond to the variants having opposite shear (self accommodating variants) in order to form twins during $t \rightarrow m$ transformation. So, a single order parameter $\phi$ represents a group of phases consisting of variant $m_{+}$, its counter self accommodating variant $m_{-}$and the parent phase $\mathrm{t}$ in $t \rightarrow m$ transformation. As discussed in the work of Levitas et al. [15] the most opted $2-4-6$ polynomial for such a crystal set is used further to describe

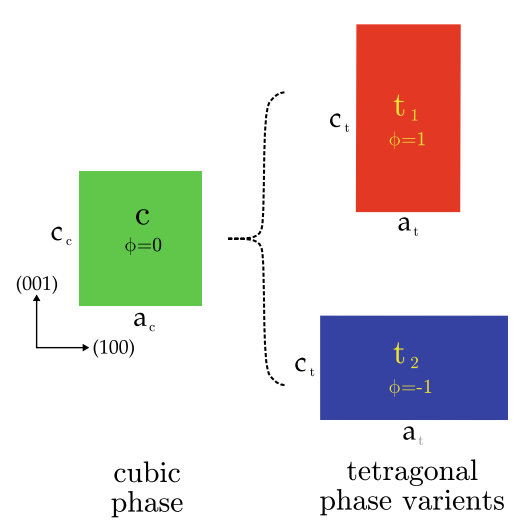

(a) $c \rightarrow t$

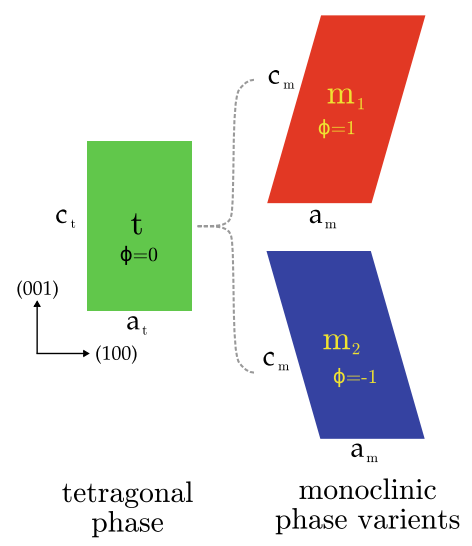

(b) $t \rightarrow m$

Fig. 21.3 Order parameter and lattice transformation representation of parent and product phases during martensitic phase transformation in zirconia ceramics. a $c \rightarrow t$ lattice transformation, $\mathbf{b}$ $t \rightarrow m$ lattice transformation schematic in two dimensions 
the potential in this work and to model the phase transformation. A similar method is considered in representing parent $c$-phase and product variants of $t$-phase during $c \rightarrow t$ transformation.

In mechanics total strain tensor is decomposed into an elastic and an inelastic/transformation term, such as,

$$
\boldsymbol{\epsilon}=\boldsymbol{\epsilon}_{\mathrm{el}}+\boldsymbol{\epsilon}_{\mathrm{tr}}(\tilde{\phi})
$$

Here $\boldsymbol{\epsilon}_{\mathrm{tr}}(\tilde{\phi})$ provides resultant transformation strain weighted with fraction of product phase $\varphi(\tilde{\phi})$. The modified order parameter $\tilde{\phi}$ is used instead of $\phi$ to avoid any unbounded solution which may lead to unphysical mechanical properties. This implies $\epsilon_{\mathrm{tr}}(0)=0$ and $\epsilon_{\mathrm{tr}}( \pm 1)=\epsilon_{\mathrm{tr}}^{m \pm}$ respectively. In same way fourth-rank effective elastic constants $\boldsymbol{E}_{\text {eff }}$ can be written as fraction weighted with respect to product phase. The transformation/Bain strains and elastic constants are listed in Tables 21.1 and 21.2. For more detailed explanation on the PF method applied especially to simulation of zirconia material we refer to our previous work [16].

The Ginzburg-Landau equations are coupled to the basic equations of continuum mechanics by applying the week form of equilibrium of momentum in a FEM framework.

$$
\nabla \cdot \sigma=0
$$

The resulting second order partial differential equations for phase-field variable $\phi$ and displacement vector $u_{i}$ are solved concurrently using the finite element method framework implemented in COMSOL multiphysics.

\subsubsection{Model Setup}

Since both the transformations $c \rightarrow t$ and $t \rightarrow m$ are martensitic, the aforementioned PF approach is used to describe both phase transformation scenarios in zirconia ceramics. It is known that, $t$-phase and $m$-phase crystal during $t \rightarrow m$ transformation share same crystal lattice points in a symmetrical manner leading to 12 possible orientation relations of parent lattice to product lattice in three dimensions (3 correspondences based on choice of lattice axis direction, two variants for each correspondence and two orientations for each variant). This is also similar to the case of $c$-phase and $t$-phase crystal during $c \rightarrow t$ transformation. In two dimensions the problem reduces to a simple set of two product crystal variants (see Fig. 21.3). The other cases of crystal transformation belong on the third dimension, so for simplification, they could be neglected from modeling. In $t \rightarrow m$ transformation this simple crystal lattice transformation set (see Fig. 21.3b) is enough to describe twin formation during transformation in two dimensions and thus could reproduce the effect of self accommodation in order to reduce the total strain energy of the system.

Since the specific domain setup differs in some cases of our simulations provided in the following subsections, they are discussed in detail in the corresponding 
Table 21.1 Variant transformation strains involved in $c \rightarrow t$ and $t \rightarrow m$ transformation $[8,17,18]$

\begin{tabular}{l|l}
\hline Lattice transformation & Transformation strain $\boldsymbol{\epsilon}_{i j}$ \\
\hline$c \rightarrow t_{1}$ & $\boldsymbol{\epsilon}_{\mathrm{tr}}^{t 1}=\left[\begin{array}{cc}-0.0007 & 0 \\
0 & 0.0197\end{array}\right]$ \\
\hline$t \rightarrow t_{2}$ & $\boldsymbol{\epsilon}_{\mathrm{tr}}^{t 2}=\left[\begin{array}{cc}0.0197 & 0 \\
0 & -0.0007\end{array}\right]$ \\
\hline$t \rightarrow m_{+}$ & $\epsilon_{\mathrm{tr}}^{m+}=\left[\begin{array}{cc}0.012479 & 0.079614 \\
0.079614 & 0.019139\end{array}\right]$ \\
\hline
\end{tabular}

Table 21.2 Elastic stiffness (in GPa) of $c$-phase, $t$-phase and $m$-phase [19]

\begin{tabular}{l|l|l|l|r|l|r|r|r|r|r|r|r|r}
\hline Phases & $\mathbf{E}_{11}$ & $\mathbf{E}_{22}$ & $\mathbf{E}_{33}$ & $\mathbf{E}_{44}$ & $\mathbf{E}_{55}$ & $\mathbf{E}_{66}$ & $\mathbf{E}_{12}$ & $\mathbf{E}_{13}$ & $\mathbf{E}_{16}$ & $\mathbf{E}_{23}$ & $\mathbf{E}_{26}$ & $\mathbf{E}_{36}$ & $\mathbf{E}_{45}$ \\
\hline$c$-phase & 390 & 390 & 390 & 60 & 60 & 60 & 162 & 162 & 0 & 162 & 0 & 0 & 0 \\
\hline$t$-phase & 327 & 327 & 264 & 59 & 59 & 59 & 100 & 62 & 0 & 62 & 0 & 0 & 0 \\
\hline$m$-phase & 361 & 408 & 258 & 100 & 81 & 126 & 142 & 55 & -21 & 196 & 31 & -18 & -23 \\
\hline
\end{tabular}

subsections of selected results. Overall in common, we assume anisotropic elastic behaviour in both elastic and phase transformation domain in our simulations. The effective transformation strain and elastic constants at a material point inside the phase transformation domain are evaluated as a function of $\phi$ [16]. The description of anisotropy is necessary to capture variant orientation relationship and the effects of various external loading directions on MPT. The material parameters used in the model are listed in Tables 21.1 and 21.2.

\subsubsection{Selected Results and Discussion}

\subsubsection{Phase Stability and Energy Barriers}

Using CALPHAD [20] method an unambiguous representation of the temperature dependent Gibbs free energy values of individual phases from the thermodynamical aspect could be evaluated [21]. But the form of energy landscape for intermediate phase transition from parent to product phase is still ambiguous and is a missing piece of puzzle. Most of the phase-field methods rely on these energy landscape to reproduce a more relevant and accurate material behavior. There are two major methods used by phase-field to approximate the transformation path a common tangent 
method [20] which expresses a linear behavior between parent and product phase minima. On other hand potential function methods [13, 22] utilize an non-linear analytical representation of path between parent and product phase minima. Both of these methods confide in phenomenological modeling of free energy based on the phase stability conditions. In this work a generic model for martensitic phase transformation developed by Levitas and Preston [14] is used and adapted for simulating zirconia ceramics material. We use $2-4-6$ potential function [16] method which is utilized to represent a non-linear behavior and exhibit a transition barrier based on thermo-mechanical conditions.

On the thermodynamic perspective of zirconia at ambient temperature, it is clear that the global minimum is at $m$-phase and the global maximum is at $t$-phase. So theoretically the metastable $t$-phase always tends to transform to stable $m$-phase. But in almost all commercial PSZ ceramics (refer $\mathrm{MgO}-\mathrm{ZrO}_{2}$ micrographs from the book of Hannink et al. [18]) the $t$-phase is observed to be stable at ambient temperature. Multiple factors may cause such a stabilization, which include: stabilizer doping such as $\mathrm{MgO}$, presence of residual stresses from prior $c \rightarrow t$ transformation, but also defects like dislocations and grain boundaries. Later in this work, the effect of residual stress is investigated. Here in this section we compare the potential functions from Mamivand et al. [11] and Levitas and Preston [14] commonly used in literature for modelling $t \rightarrow m$ transformations. We investigate the capabilities of these functions for such a stabilization.

The 2-4-6 potential used by Mamivand et al. [11] for approximating the Gibbs energy contribution defines energy barrier just by the analytical function. The barrier is then levered by the enthalpy difference between the parent and product phase irrespective of temperature, see Fig. 21.4a. Even at ambient temperature the approximated Gibbs energy landscape provides an energy barrier considering only thermodynamic contribution by pure zirconia, which is in contradiction to true physical behaviour. In the case of Levitas and Preston [14] based formulation for temperatures below $M_{s}$ the function doesn't exhibit any barrier for transformation.

At high temperature just above $M_{s}$ (see Fig. 21.4d) the energy landscape calculated based on Mamivand's potential formulation shows local minima at the parent and product phase. For the same, a global maximum or energy barrier is visible at order parameter $\phi \approx 0.1$ (see $T<M_{S}$ ). But as the temperature increases this decreases the barrier, and after crossing the $T_{0}$ there remains an intermediate local minimum which is neither parent nor product phase, the local minimum is close to order parameter $\phi \approx 0.1$, see Fig. 21.4b, c. In contrast, Levitas type potential used in this work has no intermediate minimum rather provides a barrier between the parent and product phase for temperatures above $M_{s}$.

Based on Levitas et al. formulation, utilized in this work for zirconia ceramics at any temperature, a global/local minima is retained at the product phase. And for $T>M_{s}$ a local minimum is also retained in parent phase, see Fig. $21.4 \mathrm{~b}$, d. Thus the potential function used in this work results in a proper representation of zirconia ceramics material behaviour from a pure thermodynamic stand point. 


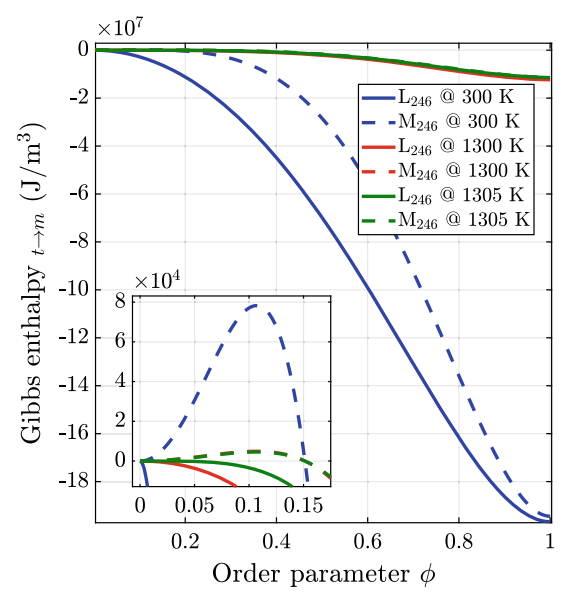

(a) @ $\mathrm{T} \leq \mathrm{M}_{s}$

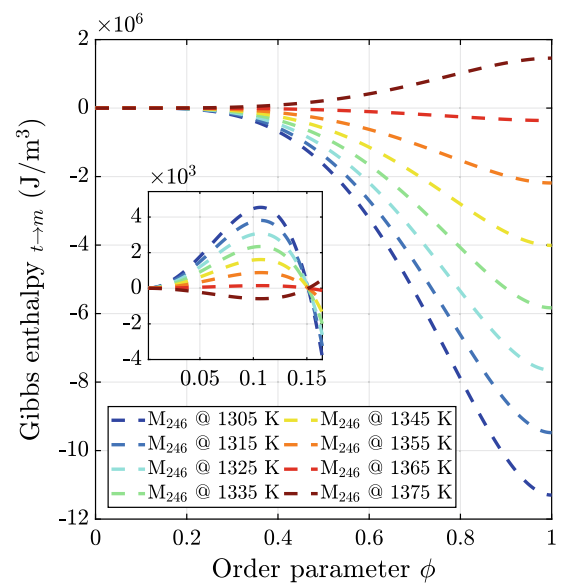

(c) Mamivand @ $\mathrm{T} \geq \mathrm{M}_{s}$

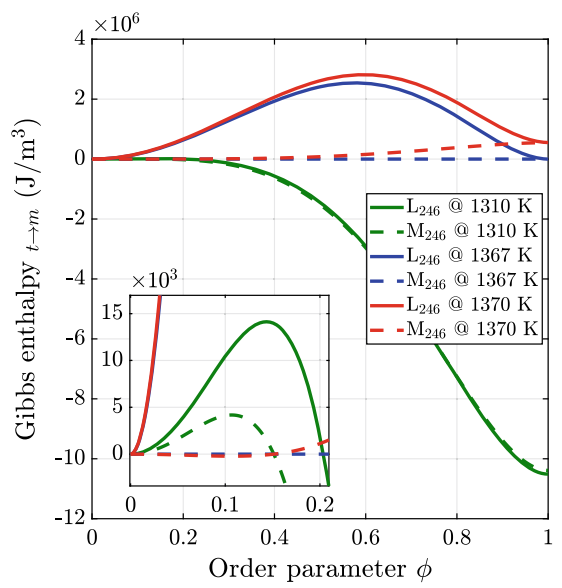

(b) @ $\mathrm{T}>\mathrm{M}_{s}$

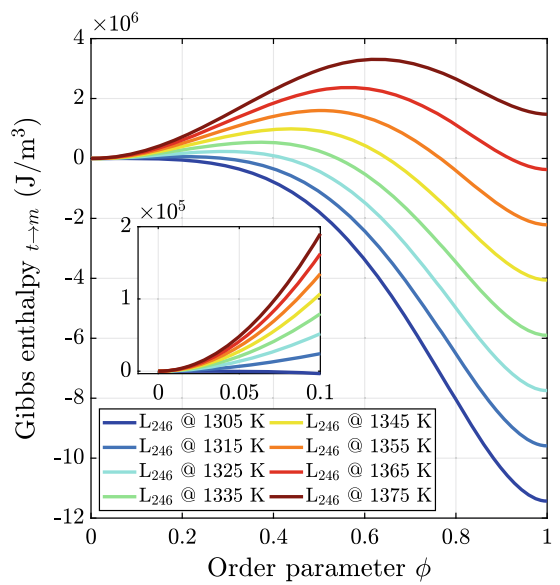

(d) Levitas $246 @ \mathrm{~T} \geq \mathrm{M}_{s}$

Fig. 21.4 Comparison of estimated 2-4-6 potential based thermodynamic Gibbs enthalpy $\psi_{\mathrm{ch}}(\theta, \phi)$ as a function of order parameter during $t \rightarrow m$ transformation at various temperatures for Levitas and Preston [14] based model used for zirconia ceramics [16] to Mamivand et al. [11] model

\subsubsection{Variant Selection by Energy Barriers}

In this section we would like present distinctive differences in microstructure and evolution path between cooling induced and stress induced $t \rightarrow m$ transformations. We show that the different behaviour can be explained by the presence of an energy barrier in the Gibbs free enthalpy. In the latter case, sequential growth of monoclinic lamellae is observed because of possible variant selection based on energy barriers, 


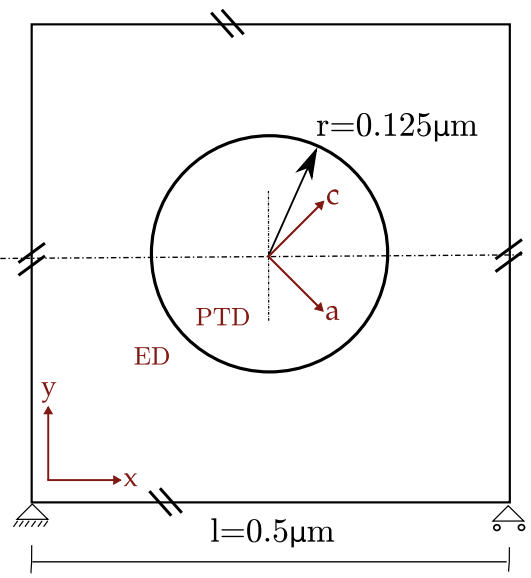

(a) Simulation domain setup

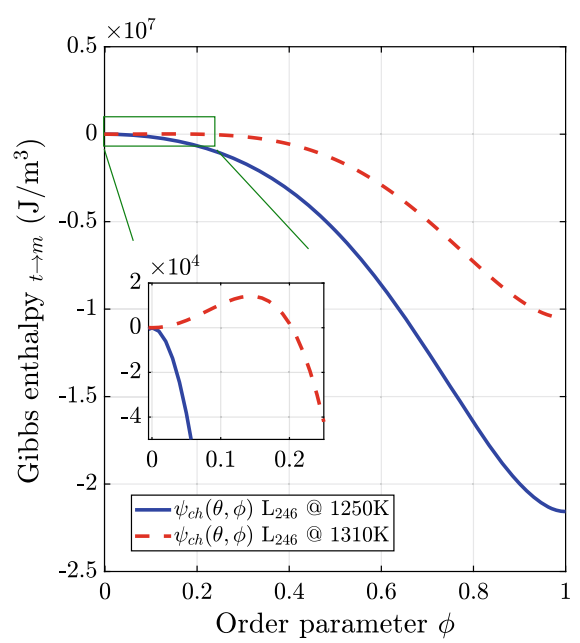

(b) $\psi_{\mathrm{ch}}(\theta, \phi) @ 1250 \mathrm{~K}$ and $1310 \mathrm{~K}$

Fig. 21.5 a Representation of simulation domain with boundary conditions. Material coordinate system (abc) and geometrical coordinate system (xyz). Axis $\mathrm{c}$ and $\mathrm{z}$ are outward normal to the paper. b Comparison of stress-independent chemical free enthalpy

whereas cooling induced microstructure evolution is characterized by an almost homogeneous nucleation of the monoclinic phase.

For simulating different microstructure evolution, such a domain setup is chosen, where the $t$-phase lentils are stabilized in the cubic matrix after annealing. A single crystal setup with a square $c$-phase elastic domain (ED) of $0.5 \mu \mathrm{m} \times 0.5$ $\mu \mathrm{m}$ with an embedded circular phase transformation domain (PTD) of radius 0.125 $\mu \mathrm{m}$ (see Fig. 21.5a) is created. As initial condition in PTD the tetragonal phase is superimposed with random noise on order parameter $\phi$ within a given range. The elastic domain here represents a cubic matrix surrounding a single $t$-phase particle. A circular form of embedded phase transformation domain is chosen in order to avoid any geometrical influence on the microstructure formation. The initial displacement is set zero in the whole domain. Additionally, a displacement periodic boundary condition is imposed at the boundaries. In order to mimic a rotated crystal around the b-axis (normal to the paper) (see Fig. 21.5a) the material parameters, transformation strain and elastic constants are transformed accordingly. Other common simulation and material parameters used are already discussed above and listed in Table 21.3.

For simulating stress induced transformation, we choose homogeneous isothermal conditions at $1310 \mathrm{~K}$, above $M_{s}=1305 \mathrm{~K}$. On the other hand, for cooling induced transformation we choose homogeneous isothermal condition at $1250 \mathrm{~K}$ below $M_{s}$. The Gibbs enthalpy landscapes based on pure thermodynamic contribution for both temperatures are compared in Fig. 21.5b.

Figure 21.6a-c show a cooling induced martensitic transformation in a single crystal, rotated by $45^{\circ}$ around 'b'-axis (see Fig. 21.5a) with isothermal condition 
Table 21.3 Input parameters used in $t \rightarrow m$ simulation

\begin{tabular}{l|l|l|l}
\hline Description & Symbol & Value & Unit \\
\hline$m$-phase start temperature & $M_{s}^{t \rightarrow m}$ & 1305 & $\mathrm{~K}$ \\
\hline$m$-phase equilibrium temperature & $T_{0}^{t \rightarrow m}$ & 1367 & $\mathrm{~K}$ \\
\hline Gradient energy coefficient & $\beta$ & $5 \times 10^{-11}$ & $\mathrm{~J} / \mathrm{m}$ \\
\hline Kinetic coefficient & $L$ & 2 & $\mathrm{~m}^{3} / \mathrm{Js}$ \\
\hline Material parameter & $a$ & 6 & - \\
\hline
\end{tabular}

(a)

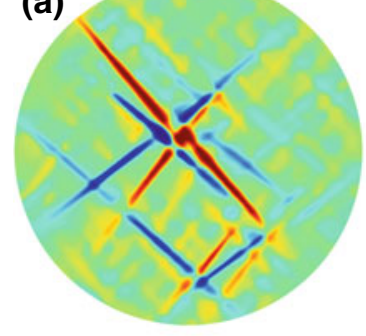

(d)

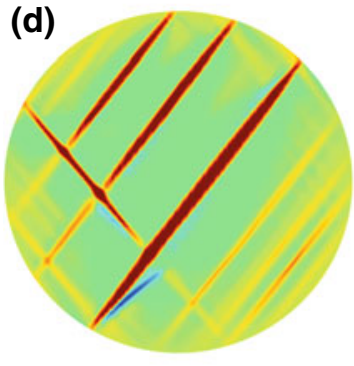

(b)

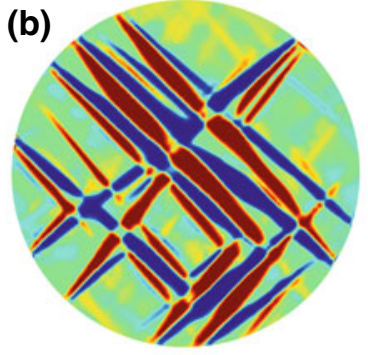

(e)

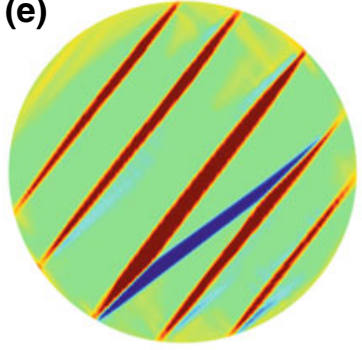

(c)

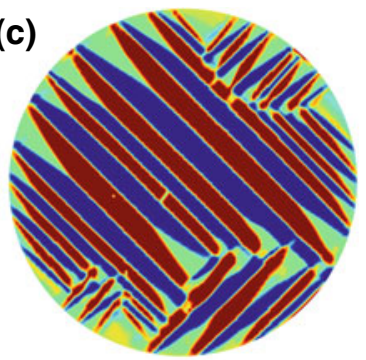

(f)

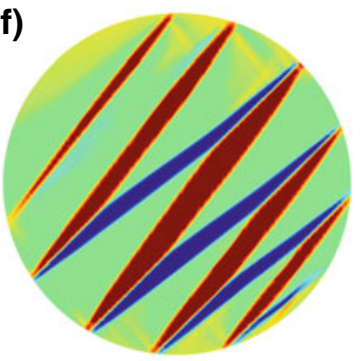

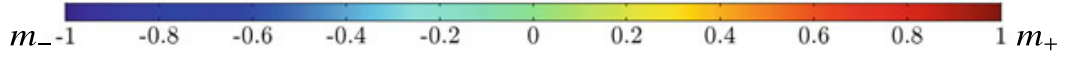

Fig. 21.6 A comparison between cooling induced case (top row $(\mathbf{a}-\mathbf{c})$ ) and stress induced case (bottom row (d-f)). The color legend represents the order parameter $\phi$. A sequential growth of lamella, observed during stress induced case due to variant selection based on external loading. Microstructure evolution snapshots at various stages of pseudo-time

below $M_{s}$ at $1250 \mathrm{~K}$. A surface plot inside the PTD shows the evolution of order parameter $\phi$ where the color legend represents, red being $m^{+}$, blue as $m^{-}$and green as $t$.

In cooling induced case there is no intermediate energy barrier between parent and product phase for transformation (see Fig. 21.5b) since the temperature is below $M_{s}$ and here product $m$-phase is stable. So after initialization an almost homogeneous nucleation process takes place where all possible nucleation sites 
of all possible variants are preferred to grow because of the adequate thermodynamic driving force (see Fig. 21.6a-d). In the numerical simulation, such condition will lead to different microstructure arrangements for different initialization. The evolved microstructures would be of mixed patterns where junction planes are parallel or orthogonal to 'c'-axis, which could be observed within a single grain (both orientation scenarios specified by Hannink et al. [18]). In Fig.21.6c a large quantity of junction plane between two $m$-phase variants are orthogonal to 'c'-axis direction and there are small amount of junction planes (upper right, lower left and lower right) oriented parallel to 'c' -axis. Figures of both such lamella directions of twin formation have already been presented in the work of Hannink et al. [18] within a single $t$-phase lentil.

Simulation parameters here remain the same as cooling induced microstructure formation case, except the operating temperature being $1310 \mathrm{~K}$ above $M_{s}$ and with $\sigma_{\text {app }}=1 \mathrm{GPa}$ compression along 'b'-axis (see Fig. 21.5a). An initial superimposed noise with a range confined within the barrier of the Gibbs enthalpy landscape (see Fig. 21.5b) is applied.

Since the pure thermodynamic driving force is not adequate to trigger the transformation, there would not be martensitic evolution at all. As the compressive stress is superimposed additionally to the thermodynamic contribution, depending on the orientation of crystal relative to the applied stress some variants are preferred to grow by decreasing the energy barrier and some are obstructed by increasing the barrier. In other words, the energy landscape is skewed such that some variants have energy barrier and others don't, see Fig. 21.9a. This becomes clear by comparison between solid blue curve where no external stress is applied, and dashed red curve after application of external stress. Here in dashed red curve one variant experiences a barrier and the other doesn't. In this example (Fig. 21.6d-f) the $m^{+}$-red nucleation sites are preferred. At the initial stage, $m^{+}$red variant nucleates and grows and meanwhile $m^{-}$ blue variant vanishes because of the energy barrier. Additionally, by superimposing normal stress $\sigma_{\text {app }}$ the driving force exceeds the minimum driving force required for transformation above $M_{s}$ and triggers the transformation. Initially only $m^{+}$variant lamellae grow such that they increase the strain energy. As the lamellae reach the grain boundary or imperfections, this piles up stress and triggers the $m^{-}$blue self accommodating variant thus reducing a part of the strain energy gained.

According to the investigation on $\mathrm{MgO}-\mathrm{ZrO}_{2}$ by Kelly and Ball [17] the potential twinning plane/junction plane for twin related variants is either $[100]_{m} /{ }^{\prime} \mathrm{a}$ '-axis or $[001]_{m} /{ }^{\prime} c$ ' -axis, based on our model base axis orientation in Fig. 21.3. The resulting junction plane $[001]_{m} /{ }^{\prime} c$ '-axis (see Fig. 21.6) is consistent with the experimental observations of $[17,18,23,24]$. It is clear that among the two possible orientation scenarios specified by Hannink et al. [18] between $m$-phase and $t$-phase, junction plane parallel to 'c'-axis, twin-related variants retain some untransformed $t$-phase , which is also consistent with our observations. But the reason for possible conditions under which such oriented structure could be reproduced has not been discussed yet before. 


\subsubsection{Origin and Effect of Residual Stresses}

The probable initial existence of residual stresses $[25,26]$ in the $t$-phase matrix as a result of the $c \rightarrow t$ transformation and their effect on $t \rightarrow m$ transformation is also not well understood yet. Such a residual stress is not yet considered in modeling for $t \rightarrow m$. In almost all commercial ceramics $t$-phase is stable at ambient temperature. Multiple factors may cause such a stabilization, here we look into the possibility and the effects of residual stress present prior to $t \rightarrow m$ transformation. In order to evaluate the peak residual stress which could be expected during $c \rightarrow t$ transformation, we simulate $c \rightarrow t$ transformation inside a $c$-phase matrix. The evolution of average pressure inside the $t$-phase lentil during transformation is tracked. This pressure is later used as an initial condition to mimic presence of residual stress during $t \rightarrow m$ transformation.

A simple model for single lentil setup within a square phase transformation domain is chosen with a size such that an average size of tetragonal lentil could be accommodated. This phase transformation domain is placed within an large elastic domain with $c$-phase . The transformation domain is initialized with $c$-phase and a circular seed of $t$-phase is placed at the centre of the phase transformation domain. The initialization and boundary conditions are set similar to those of model for $t \rightarrow m$. The domain is allowed to transform from cubic to tetragonal $(c \rightarrow t)$ by undercooling at $1300 \mathrm{~K}$ without any external mechanical loading. The thermodynamic functions for evaluating Gibbs enthalpy values are taken from [11, 21]. We assume anisotropic elastic behavior for the whole domain. The elastic constants of respective phases are provided in Table 21.2. Based on the lattice constants from [8] one can evaluate the transformation strain (see Table 21.1), the critical temperature $M_{s}^{c \rightarrow t}=1423 \mathrm{~K}$ and equilibrium temperature $T_{0}^{c \rightarrow t}=584 \mathrm{~K}$ acquired from [27]. All other parameters are similar to those of previously explained model setup and listed in Tables 21.2 and 21.3.

Figure 21.7 shows single and multi-variant $t$-phase lentils evolving inside a $c$-phase matrix. As the initial tetragonal inclusion grows to lentil shape, its interior is under compression. On left half, the mean in-plane pressure $\sigma_{\bar{p}}=-\left(\sigma_{x x}+\sigma_{y y}\right) / 2$ is plotted. The legend red represents material under compression and blue represents material under tension. On the right half, the surface plot of order parameter $\phi$ is presented. Therein, green color represents $c$-phase and red color represents $t$-phase . In commercial ceramics, stable tetragonal lentils are observed at ambient temperature. So the peak mean in-plane pressure experienced by these $t$-phase lentils during their formation is considered to be the initial condition for $t \rightarrow m$ transformation. Figure 21.8 shows an evolution of the mean in-plane pressure versus the area fraction of a single $t$-phase lentil in an infinite domain. The numerical fluctuations during the initial stages are of no interest, but a strong saturation trend in the later stages is considerd resulting in a $0.21 \mathrm{GPa}$ mean in-plane pressure inside a lentil. Although a single lentil is considered here, in reality the cubic matrix is populated with multiple lentils so a superimposed stress state of multiple lentils will lead to higher value than the one estimated here. 


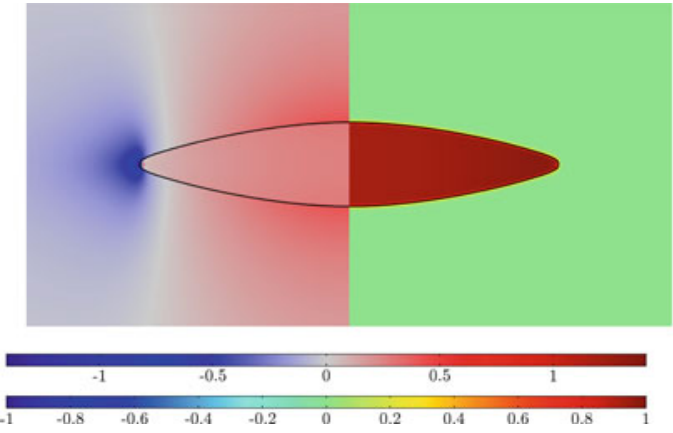

(a) Single lentil simulation

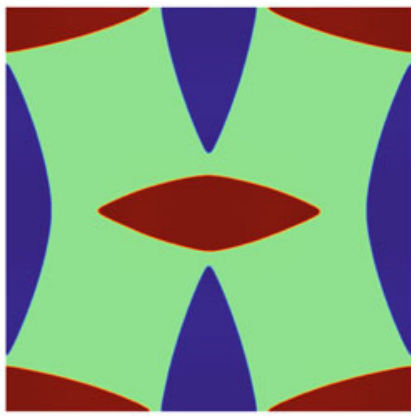

(b) Multi lentil simulation

Fig. 21.7 Evolution of single-variant and multi-variant lentils during $c \rightarrow t$ transformation

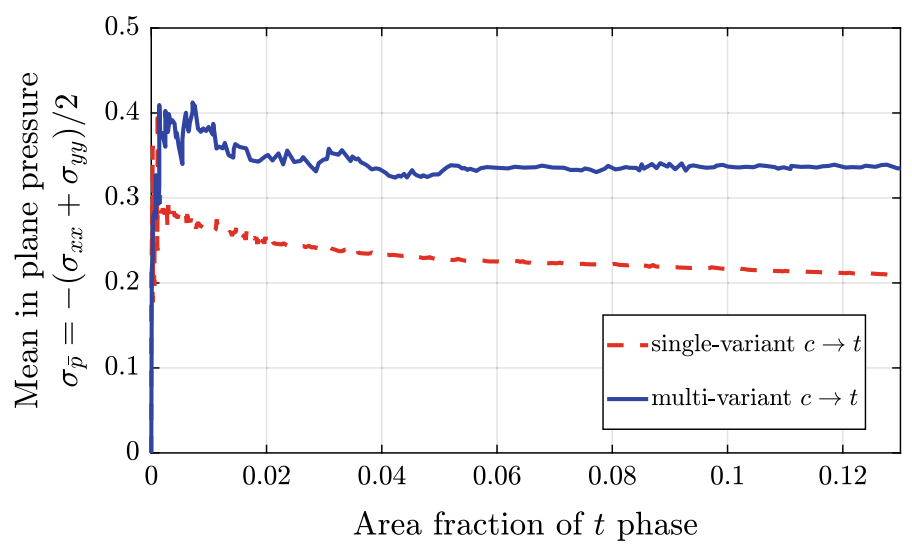

Fig. 21.8 Evolution of mean inplane pressure $\sigma_{\bar{p}}$ inside $t$-phase lentil versus fraction of $t$-phase

In order to evaluate the mean in-plane pressure on a multi lentil setup, a similar setup like a single lentil setup is choose. This setup represents a periodically placed RVE. The placement of the initial seeds are arranged such that they represent a proper microstructure. The seed at the center is replaced with a noise where an equal possibility is given to both variants to nucleate and grow. Because of the stress state of the neighboring $t$-phase lentils a selective nucleation of red variant takes place which is more favorable. The peak average pressure experienced by these $t$-phase lentils are tracked and plotted in Fig. 21.8. In this multi lentil setup the resulting mean in-plane pressure inside the lentils is $0.35 \mathrm{GPa}$ which is larger than that of the single lentil simulation case. This gives a clear evidence of residual stress from prior $c \rightarrow t$ transformation.

As consequence of result from single and multi lentil simulations we choose $\approx 0.3 \mathrm{GPa}$ as the initial pressure inside the lentil which is also consistent with the FEM based investigation on tetragonal inclusion in a cubic matrix by [26]. 
By applying a hydrostatic residual stress to the system an energy barrier is introduced thus allowing $t$-phase stability below $M_{s}$. This is visible in Fig. 21.9a on the solid blue line showing a local maximum near to the $t$-phase. The dotted green line shows the pure stress free chemical contribution of Gibbs enthalpy where the $m$-phase is stable and there is no energy barrier for $t \rightarrow m$ transformation. By superimposing an externally applied compressive stress one could skew the energy landscape, thus favouring a single variant so that stress induced transformation is possible below $M_{s}$ temperature. A similar simulation setup for $t \rightarrow m$ transformation as described in the previous section is used. Additionally, for introducing a residual stress an equibiaxial type loading of $\sigma_{\bar{p}}=-0.3 \mathrm{GPa}$ is applied. The temperature is $T=1250 \mathrm{~K}<M_{s}$. Figure $21.9 \mathrm{~b}$ shows the microstructure formed by stress induced transformation at $1250 \mathrm{~K}$. It is clear that the residual stress from the $c \rightarrow t$ transformation contributed to the stability of $t$-phase. As the operating temperature decreases the residual stress required to introduce a barrier for transformation increases (see Fig. 21.10). By this it becomes clear that residual stress is not the only contribution involved in the $t$-phase stability.

(a)

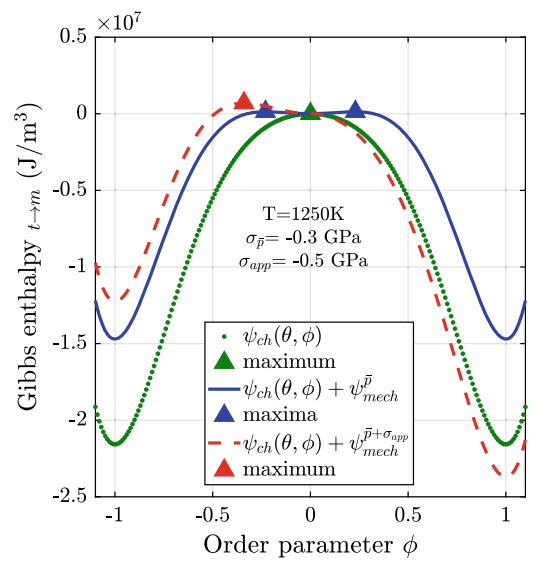

(b)

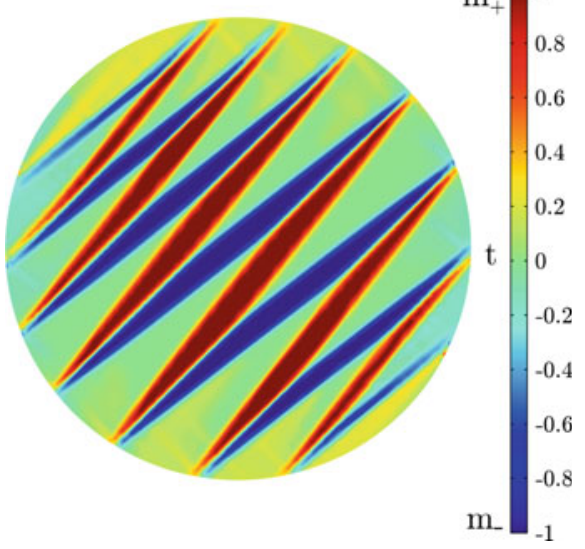

Fig. 21.9 Possibility of $t$-phase stability and stress induced transformation below $M_{S}$ temperature at $1250 \mathrm{~K}$, influence of residual stress and asymmetry in energy barrier by superimposing externally applied compression. On left, effect of residual stress and applied uniaxial compression on Gibbs enthalpy landscape below $M_{s}$ at $1250 \mathrm{~K}$. On right, surface plot of order parameter $\phi$ inside PTD. a Enthalpy landscape with various energy contributions, $\mathbf{b}$ surface plot of $\phi$ 

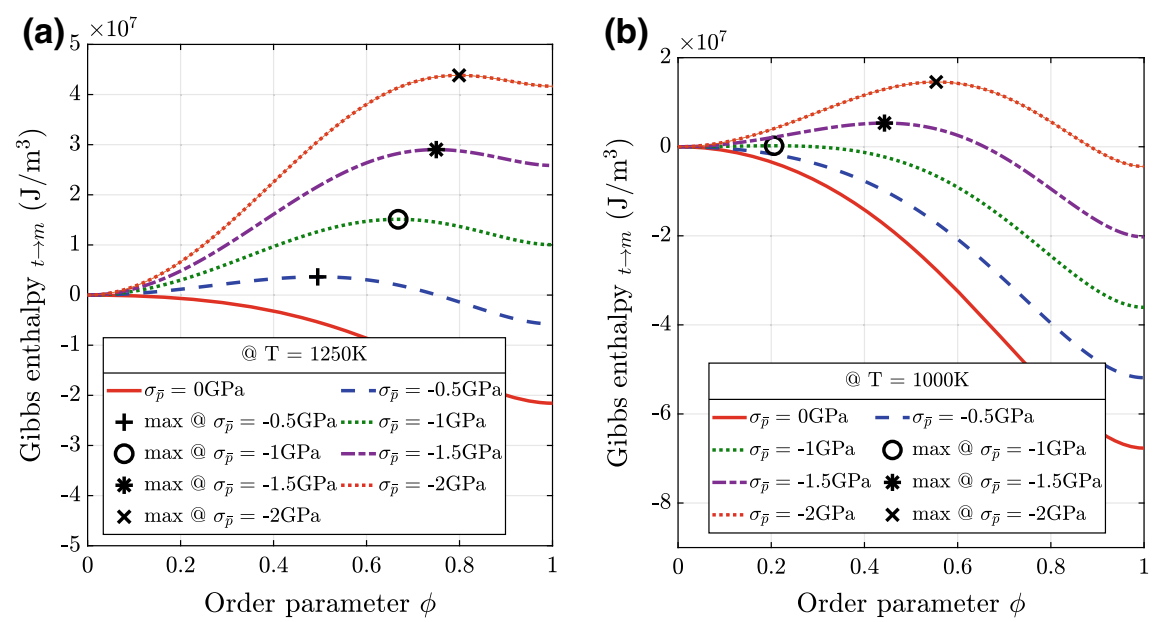

Fig. 21.10 Impact of residual stress on energy barrier at various temperatures. The intermediate local maxima showing presence of a energy barrier are represented with unique marker. A trend of increasing energy barrier with increasing residual stress is visible. a $T=1250 \mathrm{~K}, \mathbf{b} T=100 \mathrm{~K}$

\subsection{Mesomechanical Model}

The understanding of how the microstructure influences the mechanical response is an essential pre-requisite for materials tailored to match specific requirements. The aim of the present work is to develop a transformation criterion for lenticular inclusions embedded into an elastic matrix based on the work of Hensl et al. [6] that accounts for the experimentally observed tension-compression asymmetry. This criterion is then used in order to investigate the influence of the microstructural features, such as size and shape of the inclusions, on the mechanical response. A homogenization approach, schematically depicted in Fig. 21.11, provides first insights into the response of a grain.

\subsubsection{Transformation Criterion for a Single Precipitate Embedded in an Infinite Matrix}

In this section we extend the transformation criterion developed in [6] in order to account for the pressure sensitivity of the material. This is done in a plane-strain setting based on a number of assumptions. 


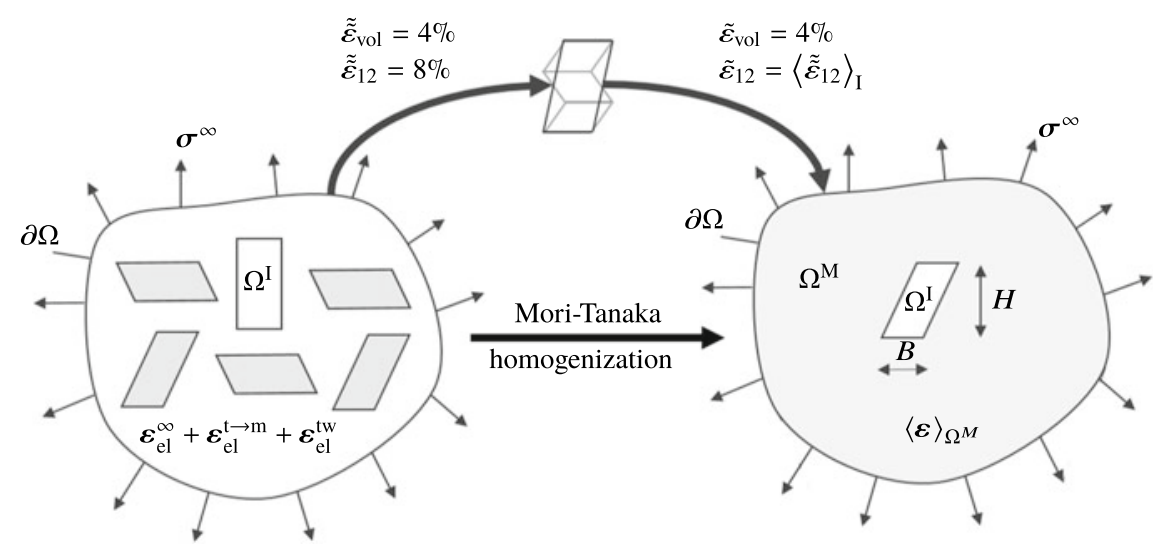

Fig. 21.11 Illustration of homogenization techniques used to model the effective material behavior of a polycrystal, influenced by the microstructure of each grain

\subsubsection{Working Hypotheses}

1. The elastic tensors of the cubic matrix and tetragonal precipitate (inclusion) are assumed to be isotropic and identical.

2. The inclusion is modeled as having a rectangular cross-section with width $B$, height $H$ and aspect ratio $\alpha=H / B$ in its untransformed state.

3. The pseudo-twin structure after $t \rightarrow m$ transformation is modeled as a stack of equal-thickness lamellae, each of which carries a strain of $\tilde{\tilde{\varepsilon}}_{11}=\tilde{\tilde{\varepsilon}}_{22}=2 \%$ resulting in a relative volume change of $\tilde{\tilde{\varepsilon}}_{\text {vol }}=4 \%$ and shear transformation strain $\tilde{\tilde{\varepsilon}}_{12}= \pm 8 \%$ (specified in the crystallographic coordinate system ${ }^{1}$ ). Note that, while all lamellae are assumed to have the same thickness, the actual value of this thickness as well as the number of lamellae $2 k$ are part of the solution and the corresponding effective shear strain of the inclusion is denoted by $\tilde{\boldsymbol{\varepsilon}}^{(k)}:=\langle\tilde{\tilde{\varepsilon}}\rangle_{\mathrm{I}}$, where $\langle\cdot\rangle_{\mathrm{I}}$ is the averaging operator over the domain of the inclusion. Specifically, $\tilde{\varepsilon}_{11}^{(k)}=\tilde{\tilde{\varepsilon}}_{11}, \tilde{\varepsilon}_{22}^{(k)}=\tilde{\tilde{\varepsilon}}_{22}$ resulting in $\tilde{\varepsilon}_{\mathrm{vol}}^{(k)}=\tilde{\tilde{\varepsilon}}_{\mathrm{vol}}=: \tilde{\varepsilon}_{\mathrm{vol}}$ and $\tilde{\varepsilon}_{12}^{(k)}=\left\langle\tilde{\tilde{\varepsilon}}_{12}\right\rangle_{\mathrm{I}}$.

4. As the specific lamellae arrangement is not part of the solution, we estimate the elastic energy contribution resulting from the "zig-zag" at the inclusion boundary by assuming strictly alternating configuration (see Fig. 21.12).

5. We assume that phase transformation occurs when the Gibbs-enthalpy is equal in the transformed $\left(G^{\mathrm{m}}\right)$ and untransformed $\left(G^{\mathrm{t}}\right)$ states, i.e.,

$$
\Delta G=G^{\mathrm{m}}-G^{\mathrm{t}}=\Delta E_{\mathrm{el}}+\Delta E_{\mathrm{ch}}+\Delta E_{\mathrm{sur}} \stackrel{!}{=} 0,
$$

\footnotetext{
${ }^{1}$ If nothing else is specified, all tensor components in this work are referred to a coordinate system with orthonormal basis $\left(O,\left\{\boldsymbol{e}_{1}, \boldsymbol{e}_{2}\right\}\right)$, where $\boldsymbol{e}_{1}$ is aligned along the tetragonal c-axis.
} 


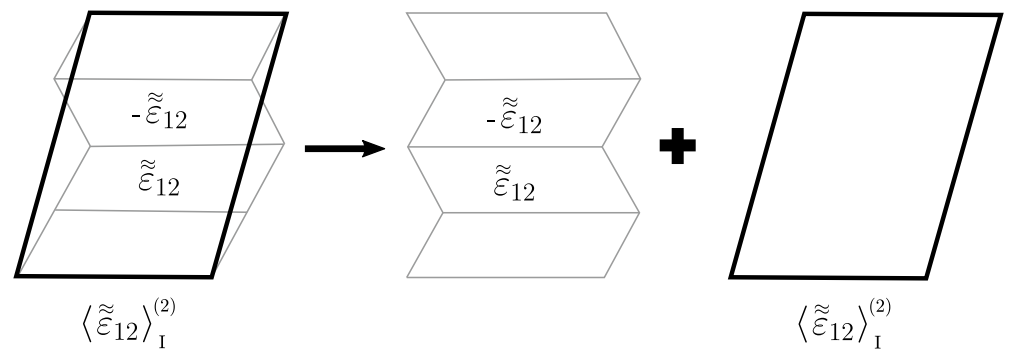

Fig. 21.12 Schematic illustration of the superposition scheme used to estimate the elastic energy of a "twinned" inclusion

where $\Delta E_{\mathrm{el}}$ is the difference in the elastic strain energy, $\Delta E_{\mathrm{ch}}$ is the difference in the chemical part of the bulk enthalpy and $\Delta E_{\text {sur }}$ is the difference in surface energy.

\subsubsection{Energetic Contributions}

The difference in surface energies is readily obtained as

$$
\Delta E_{\mathrm{sur}}=2(B+H) \Delta \beta_{\mathrm{I} / \mathrm{M}}+(2 k-1) B \beta_{\mathrm{I} / \mathrm{I}},
$$

where the first term is the contribution of the interface between inclusion and matrix and the second term corresponds to the newly formed interfaces between different monoclinic variants. Here $\beta_{\mathrm{I} / \mathrm{M}}$ is the difference in surface energies between a tetragonal-cubic and a monoclinic-cubic interface and $\beta_{\mathrm{I} / \mathrm{I}}$ is the surface energy for an interface between two different monoclinic variants. The difference in the chemical bulk enthalpy is given by

$$
\Delta E_{\mathrm{ch}}=-B H \Delta H^{t \rightarrow m} \frac{T_{0}-T}{T_{0}},
$$

where $\Delta H^{t \rightarrow m}$ is the specific transformation heat and $T_{0}$ is the equilibrium temperature between the tetragonal and monoclinic phases.

In general, the elastic strain energy is given by

$$
E_{\mathrm{el}}=\frac{1}{2} \int_{\Omega} \boldsymbol{\sigma}: \boldsymbol{\varepsilon}_{\mathrm{el}} \mathrm{d} A .
$$

In the untransformed case only the homogeneous far-field stress $\sigma^{\infty}$ and the corresponding elastic strain $\boldsymbol{\varepsilon}_{\mathrm{el}}^{\infty}=\mathbb{C}^{-1}: \sigma^{\infty}$ with elastic stiffness tensor $\mathbb{C}$ are present, i.e., 


$$
E_{\mathrm{el}}^{\mathrm{t}}=\frac{1}{2} \int_{\Omega} \boldsymbol{\sigma}^{\infty}: \boldsymbol{\varepsilon}_{\mathrm{el}}^{\infty} \mathrm{d} A=\frac{1}{2} \boldsymbol{\sigma}^{\infty}: \boldsymbol{\varepsilon}_{\mathrm{el}}^{\infty} \int_{\Omega} \mathrm{d} A .
$$

This energy is unbounded if the domain $\Omega$ is infinite. After $t \rightarrow m$ transformation and self-accommodation, additional elastic strains due to transformation $\boldsymbol{\varepsilon}_{\mathrm{el}}^{t \rightarrow m}$ and the formation of the lamellar structure $\varepsilon_{\mathrm{el}}^{\mathrm{tw}}$ are introduced, i.e.,

$$
\boldsymbol{\varepsilon}_{\mathrm{el}}=\boldsymbol{\varepsilon}_{\mathrm{el}}^{\infty}+\boldsymbol{\varepsilon}_{\mathrm{el}}^{t \rightarrow m}+\boldsymbol{\varepsilon}_{\mathrm{el}}^{\mathrm{tw}}
$$

The corresponding stress is

$$
\sigma=\sigma^{\infty}+\sigma^{t \rightarrow m}+\sigma^{\mathrm{tw}}
$$

and the energy difference between the transformed and untransformed states is immediately found to be

$$
\begin{aligned}
\Delta E_{\mathrm{el}}=E_{\mathrm{el}}^{\mathrm{m}}-E_{\mathrm{el}}^{\mathrm{t}}= & \frac{1}{2} \int_{\Omega}\left[\left(\boldsymbol{\sigma}^{\infty}+\boldsymbol{\sigma}^{t \rightarrow m}+\boldsymbol{\sigma}^{\mathrm{tw}}\right):\left(\boldsymbol{\varepsilon}_{\mathrm{el}}^{\infty}+\boldsymbol{\varepsilon}_{\mathrm{el}}^{t \rightarrow m}+\boldsymbol{\varepsilon}_{\mathrm{el}}^{\mathrm{tw}}\right)\right. \\
& \left.-\boldsymbol{\sigma}^{\infty}: \boldsymbol{\varepsilon}_{\mathrm{el}}^{\infty}\right] \mathrm{d} A
\end{aligned}
$$

Note that this difference is bounded, since the contribution of the homogeneous far-field stress cancels out and we find

$$
\begin{aligned}
\Delta E_{\mathrm{el}}= & \underbrace{\sigma^{\infty}: \int_{\Omega} \boldsymbol{\varepsilon}_{\mathrm{el}}^{t \rightarrow m} \mathrm{~d} A}_{\Delta E_{\mathrm{el}}^{\mathrm{t} \rightarrow \mathrm{m}, 1}}+\underbrace{\sigma^{\infty}: \int_{\Omega} \boldsymbol{\varepsilon}_{\mathrm{el}}^{\mathrm{tw}} \mathrm{d} A}_{\Delta E_{\mathrm{el}}^{\mathrm{tw}, 1}}+\underbrace{\frac{1}{2} \int_{\Omega} \boldsymbol{\sigma}^{t \rightarrow m}: \boldsymbol{\varepsilon}_{\mathrm{el}}^{t \rightarrow m} \mathrm{~d} A}_{\Delta E_{\mathrm{el}}^{\mathrm{t} \rightarrow \mathrm{m}, 2}}+ \\
& +\underbrace{\int_{\Omega} \boldsymbol{\sigma}^{t \rightarrow m}: \boldsymbol{\varepsilon}_{\mathrm{el}}^{\mathrm{tw}} \mathrm{d} A}_{\Delta E_{\mathrm{el}}^{\mathrm{t} \rightarrow \mathrm{tw}}}+\underbrace{\frac{1}{2} \int_{\Omega} \boldsymbol{\sigma}^{\mathrm{tw}}: \boldsymbol{\varepsilon}_{\mathrm{el}}^{\mathrm{tw}} \mathrm{d} A}_{\Delta E_{\mathrm{el}}^{\mathrm{tw}, 2}}
\end{aligned}
$$

The evalution of the individual integrals is closely related to the procedure presented in [6], which in turn is based on the closed form solution for rectangular inclusions with eigenstrains [28].

It can be shown [6] that as a particular result of assuming equal-size monoclinic lamellae, $\Delta E_{\mathrm{el}}^{\mathrm{tw}, 1}=\Delta E_{\mathrm{el}}^{\mathrm{t} \rightarrow \mathrm{tw}}=0$, while $\Delta E_{\mathrm{el}}^{\mathrm{tw}, 2}$ can be obtained in the form

$$
\Delta E_{\mathrm{el}}^{\mathrm{tw}, 2}=-B^{2} \frac{E \tilde{\tilde{\varepsilon}}_{12}^{2}}{4 \pi k^{2}\left(1-v^{2}\right)} \underbrace{\sum_{\zeta=1}^{k} \sum_{\eta=1}^{k} \sum_{i=1}^{11} g_{i}(\zeta, \eta, \alpha, k)}_{F(\alpha, k)},
$$


where the $g_{i}(\zeta, \eta)$ are functions of the geometry of the inclusion and number of lamellae, which are listed in the appendix of [6]. In order to evaluate $\Delta E_{\mathrm{el}}^{\mathrm{t} \rightarrow \mathrm{m}, 1}$ we make use of the fact that in the domain of the inclusion $\Omega^{\mathrm{I}}$ the elastic strain due to phase transformation can be computed as

$$
\boldsymbol{\varepsilon}_{\mathrm{el}}^{t \rightarrow m}=\boldsymbol{\varepsilon}^{t \rightarrow m}-\tilde{\boldsymbol{\varepsilon}}^{(k)}
$$

and can write

$$
\begin{aligned}
\Delta E_{\mathrm{el}}^{t \rightarrow m, 1} & =\sigma^{\infty}: \int_{\Omega} \boldsymbol{\varepsilon}^{t \rightarrow m} \mathrm{~d} A-\sigma^{\infty}: \tilde{\boldsymbol{\varepsilon}}^{(k)} \int_{\Omega^{\mathrm{I}}} \mathrm{d} A \\
& =\boldsymbol{\sigma}^{\infty}: \int_{\Omega} \boldsymbol{\varepsilon}^{t \rightarrow m} \mathrm{~d} A-\boldsymbol{\sigma}^{\infty}: \tilde{\boldsymbol{\varepsilon}}^{(k)} B H .
\end{aligned}
$$

Introducing the usual split into volumetric and deviatoric parts

$$
\boldsymbol{\sigma}^{\infty}=\boldsymbol{s}^{\infty}-p^{\infty} \boldsymbol{I}, \quad \boldsymbol{\varepsilon}^{t \rightarrow m}=\boldsymbol{e}^{t \rightarrow m}+\frac{1}{3} \varepsilon_{\mathrm{vol}}^{t \rightarrow m} \boldsymbol{I}, \quad \tilde{\boldsymbol{\varepsilon}}^{(k)}=\tilde{\boldsymbol{e}}^{(k)}+\frac{1}{3} \tilde{\varepsilon}_{\mathrm{vol}}^{(k)} \boldsymbol{I},
$$

where $\boldsymbol{I}$ is the unit-tensor, and making use of working Assumption 3 we find

$$
\begin{aligned}
\Delta E_{\mathrm{el}}^{t \rightarrow m, 1} & =\boldsymbol{s}^{\infty}: \int_{\Omega} \boldsymbol{e}^{t \rightarrow m} \mathrm{~d} A-p^{\infty}: \int_{\Omega} \varepsilon_{\mathrm{vol}}^{t \rightarrow m} \mathrm{~d} A+\left(p^{\infty} \tilde{\varepsilon}_{\mathrm{vol}}^{(k)}-\boldsymbol{s}^{\infty}: \tilde{\boldsymbol{e}}^{(k)}\right) B H \\
& =\boldsymbol{s}^{\infty}: \int_{\Omega} \boldsymbol{e}^{t \rightarrow m} \mathrm{~d} A-p^{\infty}: \int_{\Omega} \varepsilon_{\mathrm{vol}}^{t \rightarrow m} \mathrm{~d} A+\left(p^{\infty} \tilde{\varepsilon}_{\mathrm{vol}}^{(k)}-2 \sigma_{12}^{\infty} \tilde{\varepsilon}_{12}^{(k)}\right) B H .
\end{aligned}
$$

The evaluation of the above integrals is more involved than may appear at the first glance, since the integrands are singular at every kink at the boundary of the inclusion, therefore integration is carried out using the same procedure that was applied in [6] by a transformation into equivalent line integrals

$$
\begin{aligned}
& \Delta E_{\mathrm{el}}^{t \rightarrow m, 1}=\sigma_{12}^{\infty} \lim _{r \rightarrow \infty} \int_{0}^{2 \pi}\left[u_{1} r \sin (\varphi)+u_{2} r \cos (\varphi)\right] \mathrm{d} \varphi \\
& -p^{\infty} \lim _{r \rightarrow \infty} \int_{0}^{2 \pi}\left[u_{1} r \cos (\varphi)+u_{2} r \sin (\varphi)\right] \mathrm{d} \varphi+\left(p^{\infty} \tilde{\varepsilon}_{\mathrm{vol}}^{(k)}-2 \sigma_{12}^{\infty} \tilde{\varepsilon}_{12}^{(k)}\right) B H,
\end{aligned}
$$


resulting in the relatively simple expression

$$
\Delta E_{\mathrm{el}}^{t \rightarrow m, 1}=\frac{B H}{2(1-v)}\left[(1-2 \nu) p^{\infty} \tilde{\varepsilon}_{\mathrm{vol}}^{(k)}-\sigma_{12}^{\infty} \tilde{\varepsilon}_{12}^{(k)}\right],
$$

where $v$ is POISSON's ratio. For further details on the integration procedure, $\mathrm{cf}$. [6]. The remaining energy difference $\Delta E_{\mathrm{el}}^{t \rightarrow m, 2}$ is calculated using a very similar procedure, resulting in

$$
\Delta E_{\mathrm{el}}^{t \rightarrow m, 2}=\frac{\mu}{1-v} B H\left[P_{1}\left(\tilde{\varepsilon}_{12}^{(k)}\right)^{2}+\frac{2}{9} \frac{1-v+P_{2}}{1-2 v}\left(\tilde{\varepsilon}_{\mathrm{vol}}^{(k)}\right)^{2}\right],
$$

with

$$
\begin{aligned}
& P_{1}=\frac{1}{\pi}\left[\frac{1}{\alpha} \ln \left(1+\alpha^{2}\right)+\alpha \ln \left(1+\frac{1}{\alpha^{2}}\right)\right], \\
& P_{2}=\frac{1}{\pi}\left[\arctan \left(\frac{1}{\alpha}\right)-\arctan (\alpha)\right] .
\end{aligned}
$$

Using a basis-free representation of the stress tensor we finally find

$$
\begin{aligned}
\Delta E_{\mathrm{el}}= & \frac{B H}{2(1-v)}\left[(1-2 v) p^{\infty} \tilde{\varepsilon}_{\mathrm{vol}}^{(k)}-\left(\boldsymbol{e}_{1} \cdot \boldsymbol{\sigma}^{\infty} \cdot \boldsymbol{e}_{2}\right) \tilde{\varepsilon}_{12}^{(k)}\right]+ \\
& +\frac{\mu}{1-v} B H\left[P_{1}\left(\tilde{\varepsilon}_{12}^{(k)}\right)^{2}+\frac{2}{9} \frac{1-v+P_{2}}{1-2 v}\left(\tilde{\varepsilon}_{\mathrm{vol}}^{(k)}\right)^{2}\right] .
\end{aligned}
$$

\subsubsection{The Transformation Criterion}

Substituting the energy differences computed in the previous section into the transformation criterion (21.5) we find

$$
\begin{aligned}
\frac{1-v}{\alpha B^{2} \mu} \Delta G\left(\sigma^{\infty}, T, k\right)= & \frac{1}{2 \mu}\left[(1-2 v) p^{\infty} \tilde{\varepsilon}_{\mathrm{vol}}-\left(\boldsymbol{e}_{1} \cdot \boldsymbol{\sigma}^{\infty} \cdot \boldsymbol{e}_{2}\right) \tilde{\varepsilon}_{12}^{(k)}\right]+ \\
& +P_{1}\left(\tilde{\varepsilon}_{12}^{(k)}\right)^{2}+\frac{2}{9} \frac{1-v+P_{2}}{1-2 v} \tilde{\varepsilon}_{\mathrm{vol}}^{2}-\frac{1}{2 \pi k^{2} \alpha} \tilde{\tilde{\varepsilon}}_{12}^{2} F(\alpha, k) \\
& -\Delta H^{\mathrm{t} \rightarrow \mathrm{m}} \frac{T_{0}-T}{T_{0}} \\
& +\frac{1-v}{\mu \alpha}\left[\frac{2}{B}(\alpha+1) \Delta \beta_{\mathrm{I} / \mathrm{M}}+\frac{1}{B}(2 k-1) \beta_{\mathrm{I} / \mathrm{I}}\right] \stackrel{!}{=} 0
\end{aligned}
$$

Note that (21.26) is not an equation that can be solved directly for the stress (at fixed temperature) or temperature (at fixed stress level) required to initiate the phase 
transformation, since nearly all terms depend on the number of lamellae $2 k$. This ambiguity is resolved by choosing the number of lamellae such that it minimizes the Gibbs free enthalpy in the transformed state, i.e.,

$$
\hat{k}=\arg \min _{k \in \mathbb{N}^{+}} \Delta G\left(\sigma^{\infty}, T, k\right) .
$$

Having thus determined a value for $k$, (21.26) can be rewritten e.g. as a criterion for the applied far-field stress at fixed temperature,

$$
\left|\boldsymbol{e}_{1} \cdot \boldsymbol{\sigma}^{\infty} \cdot \boldsymbol{e}_{2}\right|-(1-2 v) \frac{\tilde{\varepsilon}_{\mathrm{vol}}}{\mid \tilde{\varepsilon}_{12}^{(\hat{k}) \mid}} p^{\infty} \stackrel{!}{=} C_{1},
$$

with

$$
\begin{aligned}
& C_{1}:=\frac{1}{\left|\tilde{\varepsilon}_{12}^{(\hat{k})}\right|}\left(2 \mu P_{1}\left(\tilde{\varepsilon}_{12}^{(k)}\right)^{2}+\frac{4 \mu}{9} \frac{1-v+P_{2}}{1-2 v} \tilde{\varepsilon}_{\mathrm{vol}}^{2}-\frac{\mu}{\pi k^{2} \alpha} \tilde{\varepsilon}_{12}^{2} F(\alpha, k)\right. \\
& \left.-2 \mu \Delta H^{t \rightarrow m} \frac{T_{0}-T}{T_{0}}+\frac{2(1-v)}{\alpha B}\left[2(\alpha+1) \Delta \beta_{\mathrm{I} / \mathrm{M}}+(2 k-1) \beta_{\mathrm{I} / \mathrm{I}}\right]\right) .
\end{aligned}
$$

For future reference we note that in the present scenario the far-field stress is at the same time the average stress over the whole domain $\langle\boldsymbol{\sigma}\rangle$ and the average matrix stress $\langle\boldsymbol{\sigma}\rangle_{\Omega^{\mathrm{M}}}$. Since in a general setting the inclusion will transform depending on the stress in the surrounding matrix, we can reinterpret the criterion (21.28) and write

$$
\left|\boldsymbol{e}_{1} \cdot\langle\boldsymbol{\sigma}\rangle_{\Omega^{\mathrm{M}}} \cdot \boldsymbol{e}_{2}\right|-(1-2 v) \frac{\tilde{\varepsilon}_{\mathrm{vol}}}{\left|\tilde{\varepsilon}_{12}(\hat{k})\right|}\langle p\rangle_{\Omega^{\mathrm{M}}} \stackrel{!}{=} C_{1} .
$$

\subsubsection{Uniaxial Loading}

In this section we apply the transformation criterion to uniaxial loading conditions in order to investigate the tension-compression asymmetry predicted by the model as well as geometric effects. 


\subsubsection{Orientation Dependence of the Transformation Stress}

In the case of uniaxial loading, i.e., $\boldsymbol{\sigma}^{\infty}=\bar{\sigma}_{11}^{\infty} \overline{\boldsymbol{e}}_{1} \otimes \overline{\boldsymbol{e}}_{1}$, we find

$$
\boldsymbol{e}_{1} \cdot \boldsymbol{\sigma}^{\infty} \cdot \boldsymbol{e}_{2}=\bar{\sigma}_{11}^{\infty}\left(\boldsymbol{e}_{1} \cdot \overline{\boldsymbol{e}}_{1}\right)\left(\overline{\boldsymbol{e}}_{1} \cdot \boldsymbol{e}_{2}\right)=\frac{1}{2} \bar{\sigma}_{11} \sin (2 \varphi) \quad \text { and } \quad p^{\infty}=-\frac{1}{3} \bar{\sigma}_{11} \text {, }
$$

where $\varphi$ is the angle enclosed between the crystallographic a-axis and the direction of loading. The transformation criterion $(21.28)$ reduces to

$$
\left|\bar{\sigma}_{11}^{\infty}\right|=\frac{C_{1}}{\frac{1}{2} \sin (2 \varphi) \pm \underbrace{\frac{1}{3}(1-2 \nu) \frac{\tilde{\varepsilon}_{\mathrm{vol}}}{\left|\tilde{\varepsilon}_{12}^{(\hat{k}) \mid}\right|}}_{=: C_{2}}}=: \sigma_{\mathrm{tr}},
$$

where the positive sign holds for tensile and the negative for compressive loading. Es expected, the stress required to initiate phase transformation strongly depends on the orientation of the inclusion relative to the applied load and is minimal if the tetragonal c-axis is aligned along the direction of maximum shear (see Fig. 21.13). Due to the increase in volume during the phase transition an asymmetry between tensile and compressive loading is observed. Further, it is clear from Fig. 21.13 that, while under sufficiently large tensile loading all inclusions will transform, this is not true in compression. In that case no transformation will occur if the misalignment between the tetragonal a-axis and the loading direction is less than $\varphi_{\text {lim }} \approx 8^{\circ}$. As a consequence, the maximal achievable transformation strain in a texture-free polycrystal is larger under tensile loading.

\subsubsection{Sensitivity with Respect to the Inclusion Size, Aspect Ratio and Interfacial Energy}

Is is clear that the critical stress to initiate phase transformation depends not only on the orientation of the inclusion relative to the applied load as discussed above, but among other parameters also on its geometry and assumptions concerning the interface energy between monoclinic lamellae. The effect of these parameters is investigated here under unaxial compression for a fixed orientation $\varphi=45^{\circ}$ by varying the values given in Table 21.4 in the range of $\pm 10 \%$. The results are shown in Figs. $21.14,21.15$ and 21.16 and concur to the expectations: The transformation stress decreases with increasing size $B$ and aspect ratio $\alpha$ of the inclusions and increases with increasing surface energy $\beta_{\mathrm{I} / \mathrm{I}}$.

The sensitivity to the inclusion size and shape is particularly pronounced; for $\alpha=5$ a change in $B$ from 29 to $24 \mathrm{~nm}$ results in a change in transformation stress 
of $250 \%$ in relative terms (see Fig.21.14). A similar effect is achieved by changing the aspect ratio from 6 to 4 (see Fig.21.15). Further, it should be noted that the transformation stress abruptly changes at certain values of $B$. This effect is due to

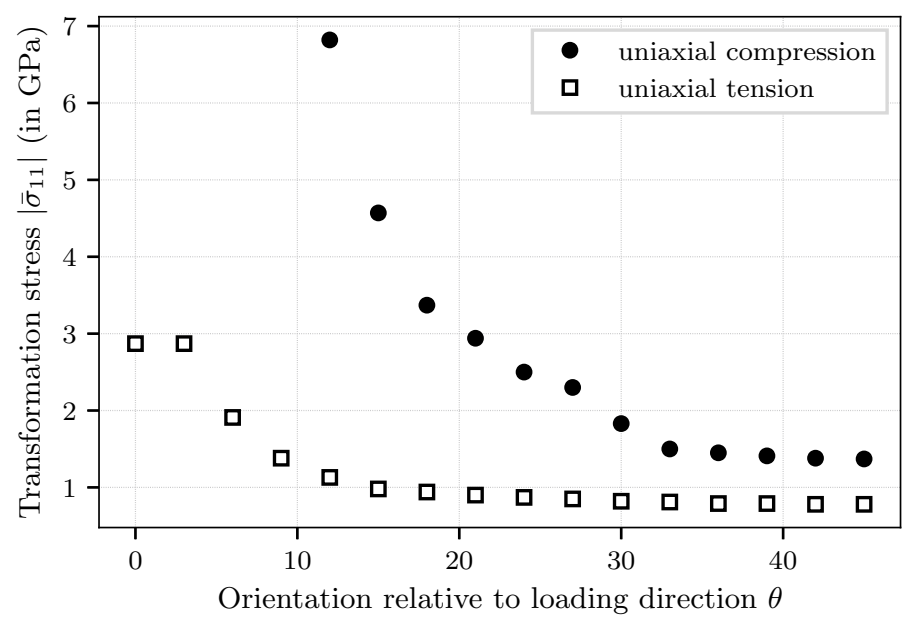

Fig. 21.13 Absolute value of the uniaxial stress necessary to induce phase transformation in dependence on the grain orientation $\varphi$ with respect to the direction of external loading

Table 21.4 Baseline for the material parameters

\begin{tabular}{l|l|l|l|l|l|l|l|l}
\hline$B$ & $\alpha$ & $E$ & $v$ & $\beta_{\mathrm{I} / \mathrm{I}}$ & $\Delta \beta_{\mathrm{I} / \mathrm{M}}$ & $\Delta H^{t \rightarrow m}$ & $T_{0}$ & $T$ \\
\hline $36 \mathrm{~nm}$ & 5.0 & $181 \mathrm{GPa}$ & 0.3 & $0.39 \mathrm{~J} / \mathrm{m}^{2}[25]$ & $0.79 \mathrm{~J} / \mathrm{m}^{2}[25]$ & $282 \mathrm{~J} / \mathrm{m}^{3}[29]$ & $1150 \mathrm{~K}[30,31]$ & $22 \mathrm{~K}$ \\
\hline
\end{tabular}

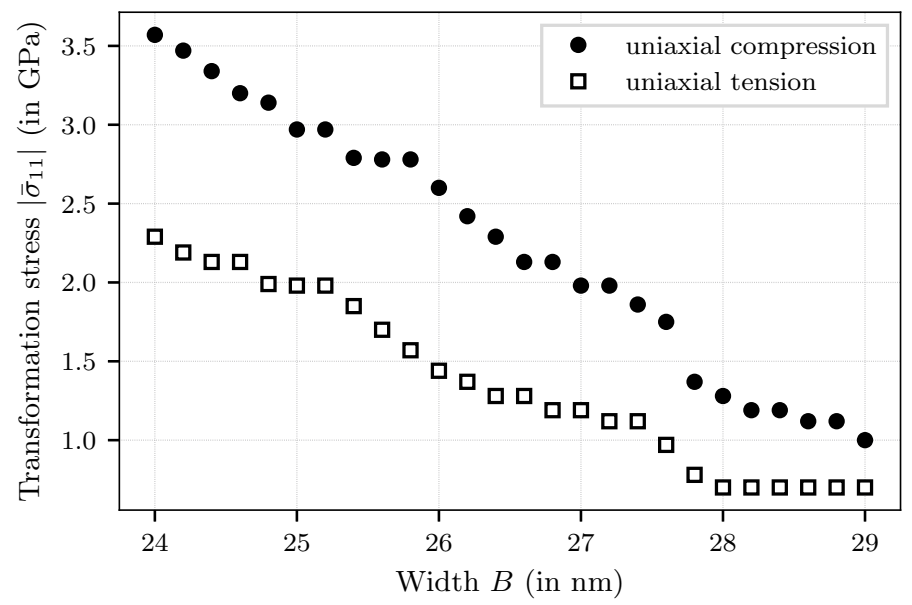

Fig. 21.14 Influence of the inclusion size $B$ on the transformation stress under uniaxial compression for inclusions oriented under $\theta=45^{\circ}$ with respect to the direction of external loading 


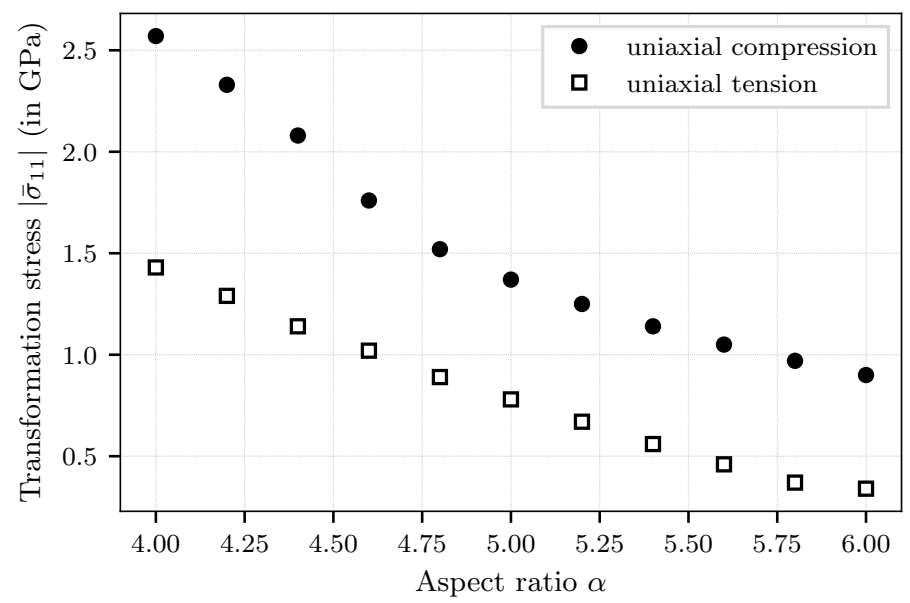

Fig. 21.15 Influence of the inclusion aspect ratio $\alpha$ on the transformation stress under uniaxial tension and compression for inclusions oriented under $\theta=45^{\circ}$ with respect to the direction of external loading

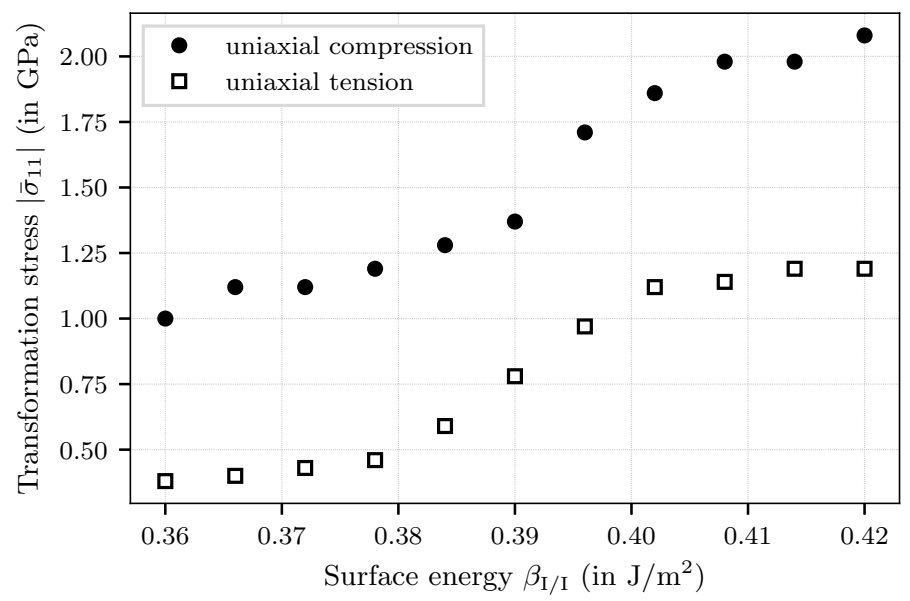

Fig. 21.16 Influence of the surface energy $\beta_{\mathrm{I} / \mathrm{I}}$ between different monoclinic variants on the transformation stress under uniaxial tension and compression for inclusions oriented under $\theta=45^{\circ}$ with respect to the direction of external loading

the discrete nature of the optimization problem (21.27) and a direct consequence of our assumptions concerning the post-transformation microstructure; every jump of the transformation stress corresponds to a change in number of lamellae $k$ and therefore to a change in microstructure. 


\subsection{Homogenization Within an Infinite Grain}

The transformation criterion developed in the previous section can be used to describe the effective mechanical response of a PSZ grain with a volume fraction $f_{\mathrm{t}}$ of tetragonal inclusions, i.e. to relate the average stress $\langle\sigma\rangle=\sigma^{\infty}$ and the average strain $\langle\boldsymbol{\varepsilon}\rangle$ via an effective elastic stiffness tensor $\langle\mathbb{C}\rangle\left(\langle\boldsymbol{\sigma}\rangle_{\mathcal{B}_{M}}, T\right)$ in the form

$$
\langle\dot{\boldsymbol{\sigma}}\rangle=\langle\mathbb{C}\rangle\left(\langle\boldsymbol{\sigma}\rangle_{\mathcal{B}_{M}}, T\right):\langle\dot{\boldsymbol{\varepsilon}}\rangle
$$

where $\langle\boldsymbol{\sigma}\rangle_{\mathcal{B}_{M}}$ is the average matrix stress, which accounts for the interaction between the inclusions in accordance with Mori-Tanaka's method. In the case of monodispersed inclusions of size $\hat{B}$ the average matrix stress is given directly by [32]

$$
\langle\boldsymbol{\sigma}\rangle_{\mathcal{B}_{M}}=\sigma^{\infty}-f_{\mathrm{m}}\left(\langle\boldsymbol{\sigma}\rangle_{\mathcal{B}_{M}}, T\right) \mathbb{C}^{\mathrm{M}}:(\mathbb{S}-\mathbb{I}): \tilde{\boldsymbol{\varepsilon}}^{(k)}\left(\langle\boldsymbol{\sigma}\rangle_{\mathcal{B}_{M}}, T, \hat{B}\right),
$$

with monoclinic volume fraction $f_{\mathrm{m}}\left(\langle\boldsymbol{\sigma}\rangle_{\mathcal{B}_{M}}, T\right)$, elastic stiffness of the matrix $\mathbb{C}^{\mathrm{M}}=$ $\mathbb{C}$ and Eshelby tensor $\mathbb{S}$. Together with the transformation criterion (21.30) this equation can be used to determine the transformation strain in the inclusions, the average matrix stress and the monoclinic phase content. As the volume expansion during $t \rightarrow m$ transformation increases the tensile stresses in the matrix, which in turn facilitate the transformation, $f_{\mathrm{m}}\left(\langle\boldsymbol{\sigma}\rangle_{\mathcal{B}_{M}}, T\right)=f_{\mathrm{t}}$ immediately after the onset of the transformation and the process is autocatalytic. The corresponding effective elastic stiffness tensor is [33]

$$
\begin{aligned}
\langle\mathbb{C}\rangle\left(\langle\boldsymbol{\sigma}\rangle_{\mathcal{B}_{M}}, T, \hat{B}\right)= & \mathbb{C}^{\mathrm{M}}+f_{\mathrm{m}}\left(\langle\boldsymbol{\sigma}\rangle_{\mathcal{B}_{M}}, T\right)\left[\mathbb{C}^{\mathrm{I}}\left(\tilde{\boldsymbol{\varepsilon}}^{(k)}\left(\langle\boldsymbol{\sigma}\rangle_{\mathcal{B}_{M}}, T, \hat{B}\right)\right)-\mathbb{C}^{\mathrm{M}}\right]: \\
& :\{\mathbb{T}\}:\left[\left(1-f_{\mathrm{m}}\left(\langle\boldsymbol{\sigma}\rangle_{\mathcal{B}_{M}}, T\right)\right) \mathbb{I}+f_{\mathrm{m}}\left(\langle\boldsymbol{\sigma}\rangle_{\mathcal{B}_{M}}, T\right)\{\mathbb{T}\}\right]^{-1}
\end{aligned}
$$

where $\mathbb{I}$ is the 4 th-order identity, $\mathbb{C}^{\mathrm{I}}\left(\tilde{\boldsymbol{\varepsilon}}^{(k)}\left(\langle\boldsymbol{\sigma}\rangle_{\mathcal{B}_{M}}, T, \hat{B}\right)\right)$ the elastic (tangent) stiffness of the inclusions,

$$
\begin{aligned}
\mathbb{T}\left(\tilde{\boldsymbol{\varepsilon}}^{(k)}\left(\langle\boldsymbol{\sigma}\rangle_{\mathcal{B}_{M}}, T, B\right)\right):= & {\left[\mathbb{I}+\mathbb{S}:\left(\mathbb{C}^{\mathrm{M}}\right)^{-1}\right.} \\
& \left.:\left[\mathbb{C}^{\mathrm{I}}\left(\tilde{\boldsymbol{\varepsilon}}^{(k)}\left(\langle\boldsymbol{\sigma}\rangle_{\mathcal{B}_{M}}, T, B\right)\right)-\mathbb{C}^{\mathrm{M}}\right]\right]^{-1}
\end{aligned}
$$

and $\{\cdot\}$ denotes the orientation average. It is well known [18] that there exists an orientation relationship between the tetragonal inclusions and the cubic parent lattice such that the principal directions of the unit cells coincide, i.e., in a two dimensional scenario two families of inclusions (denoted by subscripts $\rightarrow$ and $\uparrow$ ) with mutually orthogonal c-axes exist in each grain. As a consequence, we find 


$$
\{\mathbb{T}\}=\frac{1}{2}\left(\mathbb{T}^{\rightarrow}+\mathbb{T}^{\uparrow}\right) .
$$

Choosing an orthonormal basis such that $\boldsymbol{e}_{1}$ is aligned along the c-axis of inclusion family $\rightarrow$, we find the following non-vanishing components of the ESHELBY tensors $\mathbb{S} \rightarrow$ and $\mathbb{S}^{\uparrow}$

$$
\begin{array}{ll}
S_{1111}^{\overrightarrow{1}}=\frac{1}{2}\left[P_{1}-\left(2 P_{2}+P_{3}\right)\right], & S_{1122}^{\overrightarrow{ }}=-\frac{1}{2}\left[P_{1}+\left(2 P_{2}+P_{3}\right)\right], \\
S_{2211}^{\overrightarrow{1}}=-\frac{1}{2}\left[P_{1}-\left(2 P_{2}+P_{3}\right)\right], & S_{2222}^{\overrightarrow{1}}=\frac{1}{2}\left[P_{1}+\left(2 P_{2}+P_{3}\right)\right], \\
S_{1212}^{\overrightarrow{1}}=\frac{1}{2} P_{1}, & S_{1111}^{\uparrow}=\frac{1}{2}\left[P_{1}+\left(2 P_{2}+P_{3}\right)\right], \\
S_{1122}^{\uparrow}=-\frac{1}{2}\left[P_{1}-\left(2 P_{2}+P_{3}\right)\right], & S_{2211}^{\uparrow}=-\frac{1}{2}\left[P_{1}+\left(2 P_{2}+P_{3}\right)\right], \\
S_{2222}^{\uparrow}=\frac{1}{2}\left[P_{1}-\left(2 P_{2}+P_{3}\right)\right], & S_{1212}^{\uparrow}=\frac{1}{2} P_{1},
\end{array}
$$

with $P_{1}, P_{2}$ defined in (21.24) and

$$
P_{3}=\frac{1}{\pi}\left[\frac{1}{\alpha} \ln \left(1+\alpha^{2}\right)-\alpha \ln \left(1+\frac{1}{\alpha^{2}}\right)\right] .
$$

Prior to the $t \rightarrow m$ transformation $\mathbb{C}^{\mathrm{I}}=\mathbb{C}$ with bulk modulus $K$ and shear modulus $\mu$. To complete the formulation, assumptions concerning post-transformation behavior of $\mathbb{C}^{\mathrm{I}}$ are required, specifically

1. the elastic properties of the monoclinic and tetragonal phase are identical,

2. as long as $\left|\tilde{\varepsilon}_{12}^{(k)}\right|<\tilde{\tilde{\varepsilon}}_{12}$, the inclusions have no resistance to shear parallel to the c-axis, i.e. in the $\boldsymbol{e}_{1} \otimes \boldsymbol{e}_{2}$-direction.

As a consequence we can write the (tangent) elastic stiffness of the inclusion in Voigt notation referring to the usual crystallographic coordinate system as

$$
\mathbb{C}^{\mathrm{I}}=\left[\begin{array}{ccc}
C_{1111}^{\mathrm{I}} & C_{1122}^{\mathrm{I}} & 0 \\
C_{1122}^{\mathrm{I}} & C_{1111}^{\mathrm{I}} & 0 \\
0 & 0 & C_{1212}^{\mathrm{I}}\left(\tilde{\boldsymbol{\varepsilon}}^{(k)}\left(\langle\boldsymbol{\sigma}\rangle_{\mathcal{B}_{M}}, T, B\right)\right)
\end{array}\right]_{\boldsymbol{e}_{i} \otimes e_{j} \otimes e_{k} \otimes e_{l}}
$$

with

$$
C_{1111}^{\mathrm{I}}=K+\frac{4}{3} \mu, \quad C_{1122}^{\mathrm{I}}=K-\frac{2}{3} \mu,
$$

and 


$$
C_{1212}^{\mathrm{I}}\left(\tilde{\boldsymbol{\varepsilon}}^{(k)}\left(\langle\boldsymbol{\sigma}\rangle_{\mathcal{B}_{M}}, T, B\right)\right)= \begin{cases}\mu & \left|\tilde{\varepsilon}_{12}^{(k)}\left(\langle\boldsymbol{\sigma}\rangle_{\mathcal{B}_{M}}, T, B\right)\right|=\tilde{\tilde{\varepsilon}}_{12}, \\ 0 & \text { else. }\end{cases}
$$

Under these assumptions we obtain from (21.35)

1. before the onset of transformation $\left(f_{\mathrm{m}}\left(\langle\boldsymbol{\sigma}\rangle_{\mathcal{B}_{M}}, T\right)=0\right)$

$$
\langle\mathbb{C}\rangle\left(\langle\boldsymbol{\sigma}\rangle_{\mathcal{B}_{M}}, T, \hat{B}\right)=\mathbb{C},
$$

2. after the onset of transformation $\left(f_{\mathrm{m}}\left(\langle\boldsymbol{\sigma}\rangle_{\mathcal{B}_{M}}, T\right)=f_{\mathrm{t}}\right)$

$$
\begin{aligned}
& \langle\mathbb{C}\rangle\left(\langle\boldsymbol{\sigma}\rangle_{\mathcal{B}_{M}}, T, \hat{B}\right)=
\end{aligned}
$$

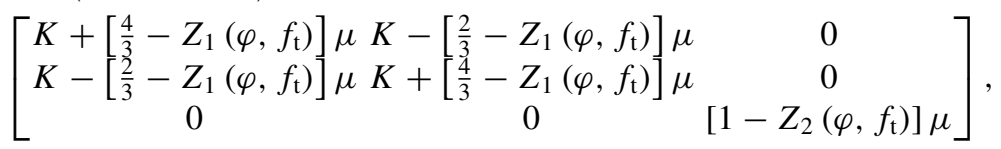

where

$$
\begin{array}{ll}
Z_{1}\left(\varphi, f_{\mathrm{t}}\right):=Z\left(f_{\mathrm{t}}\right) \sin ^{2}(2 \varphi), & Z\left(f_{\mathrm{t}}\right) \cos ^{2}(2 \varphi), \\
Z_{2}\left(\varphi, f_{\mathrm{t}}\right):=Z\left(f_{\mathrm{t}}\right)=\frac{f_{\mathrm{t}}}{1-\left[1-f_{\mathrm{t}}\right] P_{1}}, & \cos \varphi=\boldsymbol{e}_{1} \cdot \overline{\boldsymbol{e}}_{1}
\end{array}
$$

3. after the transformation shear reaches its maximum value $\tilde{\tilde{\varepsilon}}_{12}$,

$$
\langle\mathbb{C}\rangle\left(\langle\boldsymbol{\sigma}\rangle_{\mathcal{B}_{M}}, T, \hat{B}\right)=\mathbb{C} .
$$

\subsection{Continuum Mechanics Approach}

A pragmatic engineering approach to phase transition is a phenomenological modeling based on non-linear constitutive laws in the framework of continuum mechanics. The fundamentals are outlined e.g. in [34]. In particular for partially stabilized zirconia (PSZ), such a model was developed by Sun et al. [29]. Based on the concept of representative volume element (RVE) and the Hill-Rice internal variable theory [35], this model provides a set of constitutive equations for the inelastic deformations caused by tetragonal-monoclinic $t \rightarrow m$ phase transformation as function of monoclinic volume fraction. The model is restricted to a material point only. The authors of [29] did not realize an implementation of their model into a numerical tool to solve a boundary value problem for applications to real structures of PSZ. Therefore, in the present work, the Sun model was implemented into the finite ele- 
ment code ABAQUS [36] to allow simulations of the TRIP-matrix composite as will be reported in Chap. 22.

Due to missing quantitative data for the model parameters Sun et al. [29] introduced instead of this an additional hardening term in the transformation condition, which is limited to the special case of proportional mechanical loading under isothermal conditions. Another weakness of this model is the assumption and averaging of homogeneously distributed microscopic quantities over the RVE. Therefore, in Mehlhorn et al. [37] the basic concept of the Sun model has been extended to capture not only the mechanical but as well the thermally induced phase transformation and thermal expansion to simulate thermomechanical processes. Moreover, the influence of the size of transformable tetragonal particles in the cubic matrix has been incorporated. The basic assumptions and the specific formulation of the model within a thermodynamic framework will be presented in the following.

\subsubsection{Constitutive Model for Phase Transformation in PSZ}

\subsubsection{Homogenization of PSZ Material}

To find the effective material behavior, a RVE is considered with two spatially discrete components, see Fig. 21.17. The first component, called matrix, contains two crystallographic phases: the untransformable cubic zirconia and transformable, tetragonal particles embedded in the cubic phase. The second component, denoted as inclusions, contains monoclinic zirconia particles, which are generated by phase transformation from their metastable tetragonal parents when the RVE is sufficiently high loaded.

The micro-scale field quantities inside the RVE are denoted with lower case letters, such as the stress $\sigma$ and the strain $\boldsymbol{\epsilon}$. By calculating the volume average of these microscopic quantities (denoted by the operator $\langle\cdot\rangle$ ), the respective macroscopic quantities are found, which are referred to with upper case letters $\boldsymbol{\Sigma}$ and $\boldsymbol{E}$. The

Fig. 21.17 Representative volume element of PSZ: matrix of cubic and tetragonal phase, inclusions of monoclinic particles

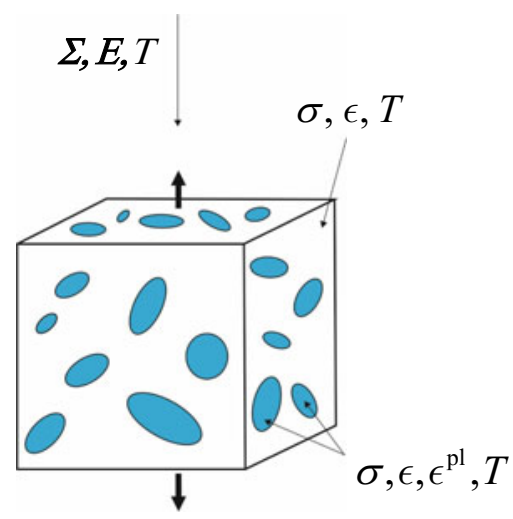


temperature $T$ is assumed to be homogeneously distributed in the RVE. We denote the RVE domain with $\mathcal{B}_{R}$, the matrix and inclusion subdomains with $\mathcal{B}_{M}$ and $\mathcal{B}_{I}$, and their volumes with $V_{R}, V_{M}$ and $V_{I}$, respectively. Thus, the relative volume fraction of transformed material is the basic internal variable calculated by

$$
f_{\mathrm{m}}=V_{I} / V_{R}
$$

The macroscopic stress tensor is obtained by volume averaging as

$$
\boldsymbol{\Sigma}=\langle\boldsymbol{\sigma}\rangle_{\mathcal{B}_{R}}=\frac{1}{V_{R}} \int_{\mathcal{B}_{R}} \boldsymbol{\sigma} \mathrm{d} V=f_{\mathrm{m}}\langle\boldsymbol{\sigma}\rangle_{\mathcal{B}_{I}}+\left(1-f_{\mathrm{m}}\right)\langle\boldsymbol{\sigma}\rangle_{\mathcal{B}_{M}} .
$$

The strain tensors can be decomposed into an elastic and plastic part $\boldsymbol{E}=\boldsymbol{E}^{\mathrm{el}}+$ $\boldsymbol{E}^{\mathrm{pl}}$ and $\boldsymbol{\epsilon}=\boldsymbol{\epsilon}^{\mathrm{el}}+\boldsymbol{\epsilon}^{\mathrm{pl}}$, respectively. Using Hooke's law, the stress-strain relation for the RVE is determined by the elastic stiffness tensor $\mathbb{C}$

$$
\boldsymbol{E}^{\mathrm{el}}=\mathbb{C}^{-1}: \boldsymbol{\Sigma}=\mathbb{C}^{-1}:\langle\boldsymbol{\sigma}\rangle_{\mathcal{B}_{R}}=\left\langle\mathbb{C}^{-1}: \boldsymbol{\sigma}\right\rangle_{\mathcal{B}_{R}}=\langle\boldsymbol{\epsilon}\rangle_{\mathcal{B}_{R}}
$$

The transformation strains exist only in the monoclinic inclusions and consist of a volumetric (dilatational) and a shear (deviatoric) component. This results in a macroscopic strain tensor

$$
\boldsymbol{E}^{\mathrm{pl}}=\boldsymbol{E}^{\mathrm{pd}}+\boldsymbol{E}^{\mathrm{ps}}=f_{\mathrm{m}}\left\langle\boldsymbol{\epsilon}^{\mathrm{pd}}\right\rangle_{\mathcal{B}_{I}}+f_{\mathrm{m}}\left\langle\boldsymbol{\epsilon}^{\mathrm{ps}}\right\rangle_{\mathcal{B}_{I}}
$$

The microscopic volume dilatation $\epsilon^{\mathrm{pd}}$ is assumed to be stress independent and constant ( $\boldsymbol{I}$ denotes the rank-two unit tensor).

$$
\boldsymbol{\epsilon}^{\mathrm{pd}}=\left\langle\epsilon^{\mathrm{pd}}\right\rangle_{\mathcal{B}_{I}}=\frac{1}{3} \operatorname{tr}\left(\boldsymbol{\epsilon}^{\mathrm{pl}}\right) \boldsymbol{I},
$$

The shear component of the transformation strain $\epsilon^{\mathrm{ps}}$, when averaged over a monoclinic volume element $d \mathcal{B}_{I}$, is proportionally related to the deviatoric stress $\boldsymbol{s}^{M}$ acting in the matrix material as follows

$$
\boldsymbol{\epsilon}^{\mathrm{ps}}=\left\langle\boldsymbol{\epsilon}^{\mathrm{ps}}\right\rangle_{d \mathcal{B}_{I}}=A \frac{\boldsymbol{s}^{M}}{\sigma_{\mathrm{eq}}^{M}}
$$

with the equivalent v.-Mises matrix stress $\sigma_{\mathrm{eq}}^{M}$. The constant material parameter $A$ describes the strength of the constraint imposed on the transformed monoclinic inclusions by the surrounding elastic matrix. The matrix stress $\sigma^{M}$ is related to the macroscopic stress $\boldsymbol{\Sigma}$ acting on the RVE, via the elastic stiffness $\mathbb{C}$ and the amount $f_{\mathrm{m}}$ of transformed phase, and can be calculated by an Eshelby approach (see e.g. [38]) and the Mori-Tanaka homogenization scheme. 
In addition, we consider a thermal expansion strain $\boldsymbol{E}^{\text {th }}$ of the RVE, whereby $\Delta T=T-T_{0}$ denotes the difference between the temperature $T$ at a specific process time and the initial or reference temperature $T_{0}$. The thermal expansion tensor $\alpha$ is taken as constant.

$$
\boldsymbol{E}^{\mathrm{th}}=\alpha \Delta T \text {. }
$$

\subsubsection{Thermodynamic State Potentials}

According to the thermodynamical framework of material modeling (see e.g. [39]), the constitutive equations of an elastic-plastic material can be derived from energy potential functions. The thermodynamical state of the RVE can be defined by the specific Helmholtz free energy $\varphi_{R}$, which is a function of the strain $\boldsymbol{E}$, the temperature $T$ and the actual state of inelastic deformation represented by the monoclinic volume fraction $f_{\mathrm{m}}$ and the transformation strain $\left\langle\epsilon^{\mathrm{ps}}\right\rangle_{\mathcal{B}_{I}}$.

$$
\varphi_{R}=\varphi_{R}\left(\boldsymbol{E}, T, f_{\mathrm{m}},\left\langle\epsilon^{\mathrm{ps}}\right\rangle_{\mathcal{B}_{I}}\right)
$$

The Helmholtz free energy $\varphi_{R}$ of PSZ consists of three components: the stored elastic energy $\varphi_{R}^{\mathrm{el}}$, the change in chemical free energy $\Delta \varphi_{R}^{\mathrm{ch}}$ and the surface free energy $\Delta \varphi_{R}^{\text {sur }}$

$$
\varphi_{R}=\varphi_{R}^{\mathrm{el}}+\Delta \varphi_{R}^{\mathrm{ch}}+\Delta \varphi_{R}^{\mathrm{sur}}
$$

The stored elastic energy $\varphi_{R}^{\mathrm{el}}$ is composed of two contributions: (i) the energy stored due to the elastic deformation, which is the total strain minus transformation and thermal strain terms $\boldsymbol{E}^{\mathrm{el}}=\boldsymbol{E}-\boldsymbol{E}^{\mathrm{pl}}-\boldsymbol{E}^{\text {th }}$, and (ii) the elastic energy stored due to the internal stresses which are induced by the transformational eigenstrains $\epsilon^{p l}$. Substituting the according expressions from (21.50) and (21.53), we get

$$
\begin{aligned}
\varphi_{R}^{\mathrm{el}}\left(\boldsymbol{E}, T, f_{\mathrm{m}},\left\langle\boldsymbol{\epsilon}^{\mathrm{pl}}\right\rangle_{\mathcal{B}_{I}}\right)= & \frac{1}{2}\left(\boldsymbol{E}-f_{\mathrm{m}}\left\langle\boldsymbol{\epsilon}^{\mathrm{pl}}\right\rangle_{\mathcal{B}_{I}}-\boldsymbol{\alpha} \Delta T\right): \mathbb{C}:\left(\boldsymbol{E}-f_{\mathrm{m}}\left\langle\boldsymbol{\epsilon}^{\mathrm{pl}}\right\rangle_{\mathcal{B}_{I}}-\boldsymbol{\alpha} \Delta T\right) \\
& -\frac{f_{\mathrm{m}}}{2 V_{R}} \int_{\mathcal{B}_{R}} \boldsymbol{\sigma}: \boldsymbol{\epsilon}^{\mathrm{pl}} \mathrm{d} V .
\end{aligned}
$$

A detailed derivation of the last term in the above equation is given in [40].

Given the difference of volume specific chemical free energy $\Delta \varphi^{\operatorname{ch}(t \rightarrow m)}$ between tetragonal and monoclinic phases of zirconia, the chemical free energy of the RVE $\Delta \varphi_{R}^{\mathrm{ch}}$ is changed during phase transformation by

$$
\Delta \varphi_{R}^{\mathrm{ch}}\left(T, f_{\mathrm{m}}\right)=f_{\mathrm{m}} \Delta \varphi^{\mathrm{ch}}(T)=f_{\mathrm{m}} q\left(\frac{T}{T^{*}}-1\right) .
$$


In the present extension of the Sun model a temperature dependence of the phase transformation is incorporated by specifying $\Delta \varphi^{\mathrm{ch}}(T)$ as a function of the temperature, where $q$ is the volume specific heat of transformation of zirconia and $T^{*}$ is the tetragonal-monoclinic equilibrium temperature of zirconia.

Due to phase transformation the interface between particles and matrix exhibits a surface free energy $\Delta \varphi_{R}^{\text {sur }}$. This energy term per volume of the RVE is calculated from the change of specific surface free energy $\Delta \varphi^{\operatorname{sur}(t \rightarrow m)}$ of zirconia and the monoclinic volume fraction $f_{\mathrm{m}}$ as follows

$$
\Delta \varphi_{R}^{\mathrm{sur}}\left(f_{\mathrm{m}}\right)=\frac{3 f_{\mathrm{m}}}{r\left(f_{\mathrm{m}}\right)} \Delta \varphi^{\mathrm{sur}(t \rightarrow m)} .
$$

In the original work [29] all transformable inclusions are assumed as spheres of equal size of radius $r\left(f_{\mathrm{m}}\right)=$ const. However, the phase stability of a particle depends on its size as shown by Garvie [25], i.e. that smaller crystallites require a higher thermodynamical driving force to transform than larger particles. Therefore, Mehlhorn et al. [37] introduced a more realistic approach by assuming a continuous size distribution of transformable particles in the model. As a first approximation, this distribution function $h(r)$ may be chosen as constant in the

$$
h(r)= \begin{cases}\frac{1}{r_{\max }-r_{\min }} & \text { for } r_{\min } \leq r \leq r_{\max } \\ 0 & \text { for } r<r_{\min } \text { or } r>r_{\max } .\end{cases}
$$

During loading, the phase transformation starts at largest particles with radius $r_{\max }$. A further increase in loading will trigger smaller particles to transform until all particles down to the radius $r_{\min }$ have become monoclinic. In the intermediate stage, the volume of all transformed particles, whose size is in the interval $\left[r, r_{\max }\right]$, is

$$
V(r)=\frac{4}{3} \pi \int_{r}^{r_{\max }} h(\bar{r}) \bar{r}^{3} \mathrm{~d} \bar{r}, \quad f_{\mathrm{m}}(r)=\frac{V(r)}{V_{R}} .
$$

constituting a corresponding volume fraction $f_{\mathrm{m}}(r)$ in the RVE. The maximum volume fraction is attained when all particles are monoclinic $f_{\mathrm{m}}^{\max }=f_{\mathrm{m}}\left(r_{\mathrm{min}}\right)$. Inserting (21.59) in (21.60) leads to

$$
f_{\mathrm{m}}(r)=\frac{1-\left(r / r_{\max }\right)^{4}}{1-\left(r_{\min } / r_{\max }\right)^{4}} f_{\mathrm{m}}^{\max }
$$

The inverse $f_{\mathrm{m}}^{-1}(r)=r\left(f_{\mathrm{m}}\right)$ can be calculated analytically as follows

$$
r\left(f_{\mathrm{m}}\right)=r_{\max }\left[1-\left(1-\left(r_{\min } / r_{\max }\right)^{4}\right) \frac{f_{\mathrm{m}}}{f_{\mathrm{m}}^{\max }}\right]^{\frac{1}{4}},
$$

which marks the particle size dependent change in surface energy in (21.58). 
By introducing a second thermodynamic dissipation function, an energetic transformation criterion was derived [29], which represents a combination of an isotropic expanding and kinematic shifting limit surface in the macroscopic stress space

$$
F\left(\boldsymbol{\Sigma}, f_{\mathrm{m}},\left\langle\boldsymbol{\epsilon}^{\mathrm{pl}}\right\rangle_{\mathcal{B}_{I}}\right)=\left(\boldsymbol{\Sigma}-f_{\mathrm{m}} \mathbb{C}:(\boldsymbol{\Lambda}-\mathbb{I}):\left\langle\boldsymbol{\epsilon}^{\mathrm{pl}}\right\rangle_{\mathcal{B}_{I}}\right):\left\langle\boldsymbol{\epsilon}^{\mathrm{pl}}\right\rangle_{d \mathcal{B}_{I}}-C\left(f_{\mathrm{m}}\right)=0 .
$$

Hereby, the average matrix eigenstress $-f_{\mathrm{m}} \mathbb{C}:(\boldsymbol{\Lambda}-\mathbb{I}):\left\langle\boldsymbol{\epsilon}^{\mathrm{pl}}\right\rangle_{\mathcal{B}_{I}}$ acts as backstress. ( $\boldsymbol{\Lambda}$ is the so-called Eshelby tensor and $\mathbb{I}$ denotes the rank-four unity tensor.) The term $C\left(f_{\mathrm{m}}\right)$ represents the energetic barrier, which must be overcome for phase transformation. It contains energy constants as well as a phenomenological hardening function depending linearly on $f_{\mathrm{m}}$. Finally, the macroscopic constitutive law is obtained as relationship between the rates of strain and stress

$$
\dot{\boldsymbol{E}}=\mathbb{C}^{-1}: \dot{\boldsymbol{\Sigma}}+\dot{f}_{\mathrm{m}}\left(\epsilon^{\mathrm{pd}} \boldsymbol{I}+A \frac{\boldsymbol{s}^{M}}{\sigma_{e q}^{M}}\right)
$$

wherein the first term represents the elastic behavior and the second the inelastic deformation due to phase transformation. The general form resembles to a rateindependent associated flow. The rate of phase change $\dot{f}_{\mathrm{m}}$ is obtained from the consistency condition $\dot{F}=0$.

This constitutive relationship is conformal with the second law of thermodynamics, demanding that the dissipation rate $\mathcal{D}$ is always positive. Obviously, the dissipation rate is proportional to the change in volume fraction of the monoclinic phase

$$
\mathcal{D}= \begin{cases}D_{0} \dot{f}_{\mathrm{m}} & \dot{f}_{\mathrm{m}}>0 \text { (tetragonal-to-monoclinic) } \\ -D_{0} \dot{f}_{\mathrm{m}} & \dot{f}_{\mathrm{m}}<0 \text { (monoclinic-to-tetragonal) }\end{cases}
$$

The proportionality factor $D_{0}$ is a phenomenological model parameter. The model accounts for both forward and reverse phase transformation by distinguishing between positive and negative rates of change. Also, the expression of $C$ differs depending on whether forward or reverse transformation occurs.

\subsubsection{Numerical Results}

\subsubsection{Particle Size Dependent Surface Energy Change}

In order to understand, how the radius $r$ of the currently active transforming particles varies with the monoclinic volume fraction $f_{\mathrm{m}}$ during the process of phase transformation, (21.62) is studied for three different size distribution functions $h_{i}\left(r_{\min }, i, r_{\max }\right)$ with $i=1,2,3$. For the upper limit of the particle size range a typical PSZ particle 
Fig. 21.18 Graphical representation of the function $r\left(f_{\mathrm{m}}\right)$ in (21.62) for different particle size ratios and $f_{\mathrm{m}}^{\max }=0.25$ [37]

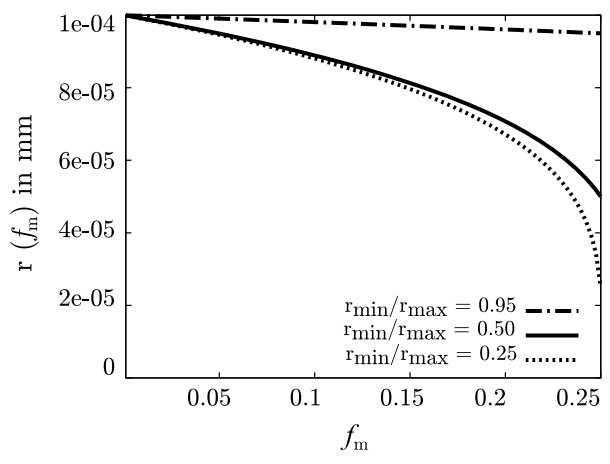

Fig. 21.19 Graphical representation of surface energy $\Delta \varphi_{R}^{\text {sur }}$ in (21.58) for different particle size ratios [37]

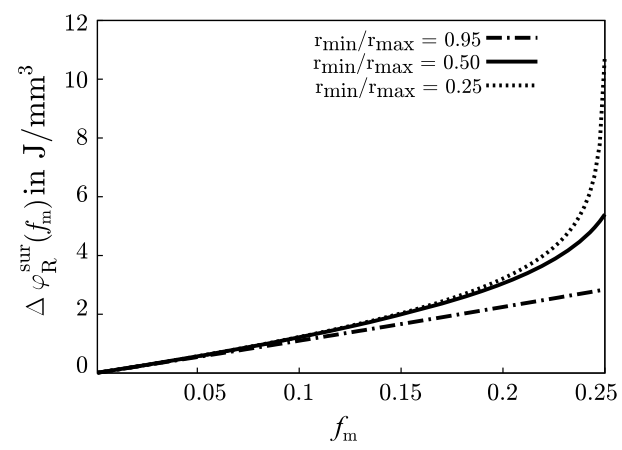

radius of $r_{\max }=1 \cdot 10^{-7} \mathrm{~m}$ is chosen. The values of $r_{\min }$ are taken as following fractions: $r_{\min , 1} / r_{\max }=0.95, r_{\min , 2} / r_{\max }=0.5$, and $r_{\min , 3} / r_{\max }=0.25$. Figure 21.18 gives a graphical representation of (21.62), using these values. It is obvious that a narrow size distribution (as $r_{\min } / r_{\max }=0.95$ ) is very close to a constant particle size of the original Sun model, resulting in a slight dependence of the radius $r$ on the transformed volume fraction $f_{\mathrm{m}}$. The wider the distribution function $h\left(f_{\mathrm{m}}\right)$ is (i.e. with smaller particle size ratios $r_{\min } / r_{\max }$ ), the stronger is the nonlinear dependence of $r$ on $f_{\mathrm{m}}$.

The influence of $r(f)$ on the volume specific surface energy change $\Delta \varphi_{R}^{\text {sur }}$ (21.58) is illustrated in Fig. 21.19. For a narrow particle size distribution with ratio $r_{\min } / r_{\max }=0.95$, the surface energy change $\Delta \varphi_{R}^{\text {sur }}$ grows almost linear with $f_{\mathrm{m}}$ similar as in the original Sun model. For smaller ratios $r_{\min } / r_{\max }$, the extended material model shows a strong nonlinear increase of $\Delta \varphi_{R}^{\text {sur }}$, especially if $f_{\mathrm{m}} \rightarrow f_{\mathrm{m}}^{\mathrm{max}}$, as it can be seen for $r_{\min } / r_{\max }=0.25$ in Fig. 21.19. This means, $\Delta \varphi_{R}^{\text {sur }}$ acts as a transformation barrier, preventing very small particles from transforming even under high thermal or mechanical loading. 


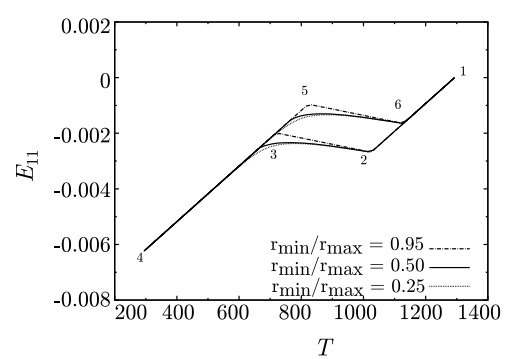

(a) Strain-temperature curves $\left(D_{0}=10 \mathrm{MPa}\right.$, $f_{\mathrm{m}}^{\max }=0.25$ )

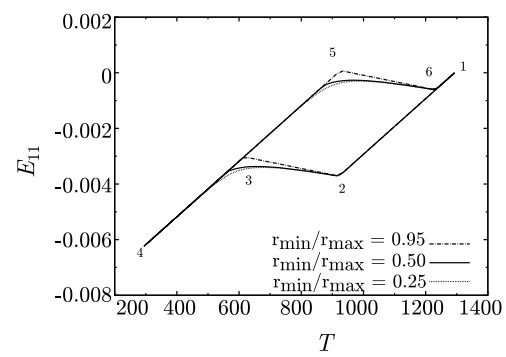

(c) Strain-temperature curves $\left(D_{0}=30 \mathrm{MPa}\right.$, $f_{\mathrm{m}}^{\max }=0.25$ )

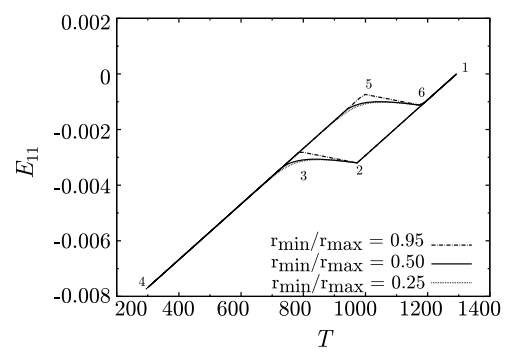

(e) Strain-temperature curves $\left(D_{0}=20 \mathrm{MPa}\right.$, $\left.f_{\mathrm{m}}^{\max }=0.15\right)$

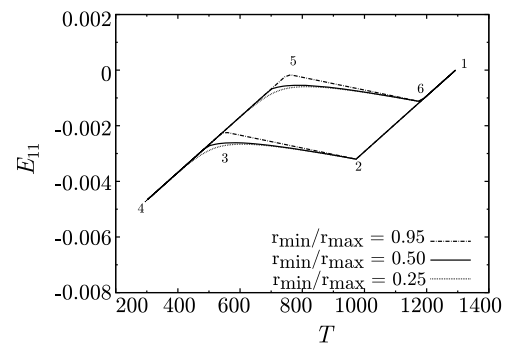

(g) Strain-temperature curves $\left(D_{0}=20 \mathrm{MPa}\right.$, $f_{\mathrm{m}}^{\max }=0.35$ )

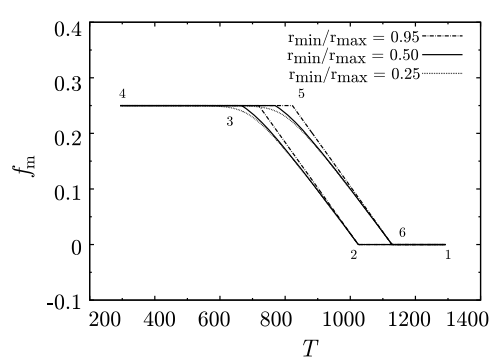

(b) Phase fraction-temperature curves $\left(D_{0}=\right.$ $10 \mathrm{MPa}, f_{\mathrm{m}}^{\max }=0.25$ )

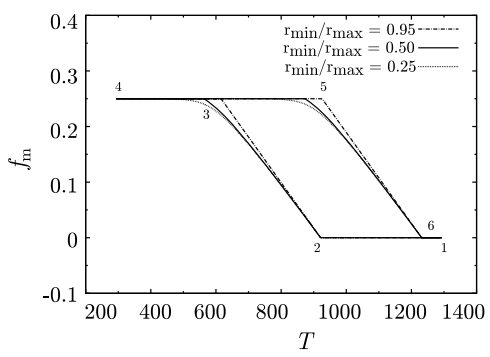

(d) Phase fraction-temperature curves $\left(D_{0}=\right.$ $30 \mathrm{MPa}, f_{\mathrm{m}}^{\max }=0.25$ )

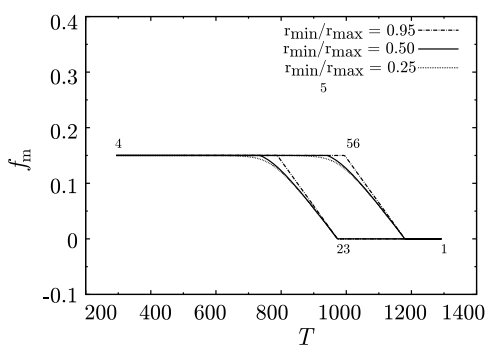

(f) Phase fraction-temperature curves $\left(D_{0}=\right.$ $20 \mathrm{MPa}, f_{\mathrm{m}}^{\max }=0.15$ )

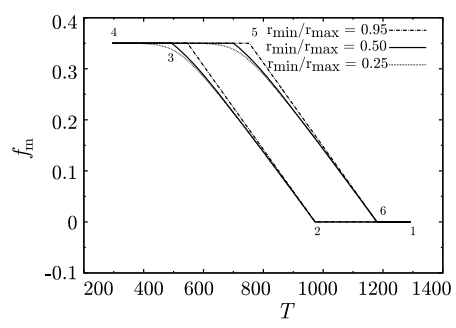

(h) Phase fraction-temperature curves $\left(D_{0}=\right.$ $20 \mathrm{MPa}, f_{\mathrm{m}}^{\max }=0.35$ )

Fig. 21.20 Calculated strain-temperature curves and corresponding phase development for different particle size ratios. The sequence of the cooling-heating cycle is visualized by the numbers $1, \ldots, 6[37]$ 


\subsubsection{Temperature-Induced Phase Transformation}

In order to demonstrate the ability of the extended material model to reproduce the hysteresis strain-temperature behavior of PSZ ceramics, a cooling-heating cycle $1373 \mathrm{~K}-293 \mathrm{~K}-1373 \mathrm{~K}$ is numerically simulated. The required model parameters associated with the changes in chemical energy ( $q$ and $T^{*}$, see (21.57)), are taken from literature: heat of transformation $q=2.82 \mathrm{~J} / \mathrm{m}^{3}$ and the phase equilibrium temperature $T^{*}=1447 \mathrm{~K}$. The difference in specific surface energies between the tetragonal and monoclinic phase was set to $\Delta \varphi^{\operatorname{sur}(t \rightarrow m)}=0.36 \mathrm{~J} / \mathrm{m}^{2}$, see [37]. Since no values for the dissipation parameter $D_{0}$ and the amount of transformable tetragonal material $f_{\mathrm{m}}^{\max }$ were available, they were estimated in order to obtain physically meaningful results. Moreover, a variation of these parameters is performed to study their influence on the material model behavior. $D_{0}$ was specified to the values 10,20 , and $30 \mathrm{MPa}$. $f_{\mathrm{m}}^{\max }$ was set to $0.15,0.25$ and 0.35 , respectively. For all remaining model parameters the values published by Sun et al. [29] are used.

Figure 21.20 shows the numerically obtained strain-temperature curves $E_{11}-T$ and the corresponding phase evolution $f_{\mathrm{m}}-T$ during the cooling-heating cycle for different sets of model parameters. As it can be seen, the typical strain hysteresis loops of PSZ ceramics are predicted by the material model, caused by a tetragonalto-monoclinic transformation on cooling and a reverse transformation on heating. In each diagram, the influence of particle size distribution is included by varying the ratio $\left(r_{\min , i}, r_{\max }\right)$. It can be seen in all diagrams, that smaller size ratios lead to a considerably nonlinear strain-temperature behavior and rounded transition curves. The influence of $D_{0}$ on the strain-temperature curves can be observed in Fig. 21.20a-d. $D_{0}$ governs the size of the strain hysteresis between cooling and heating. In contrast, $f_{\mathrm{m}}^{\max }$ influences the total transformation strain and hence the length of the temperature interval in which transformation occurs, see Fig. 21.20e-h.

These results demonstrate the feasibility of the extended material model, which forms a solid basis for simulations of structures and composites made of PSZ. Unfortunately, it was not possible to identify the required parameters for the type of $\mathrm{MgO}$ stabilized $\mathrm{ZrO}_{2}$ manufactured in the CRC799.

\subsection{Simulations of $\mathrm{ZrO}_{2}$-Particle Reinforced TRIP-Steel Composite}

In order to assist the development of particle reinforced composites manufactured by a powder metallurgical process route from TRIP-steel and partially stabilized $\mathrm{ZrO}_{2}$ ceramics particles, accompanying numerical simulations have been carried out. The mechanical properties of such a composite material are quite complex as they arise from the properties of its individual components, their volume content, and the properties of the interface between them. As explained in the previous sections, PSZ can undergo a stress-triggered phase transformation. This can lead to an additional 
toughening effect compared to non-transformable ceramics as observed in [41]. The TRIP-steel exhibits a deformation induced phase transformation from the austenitic parent phase to martensite. By combining the two materials using TRIP steel as matrix and PSZ as strengthening particles, an elasto-viscoplastic particle-reinforced composite is created with the capability of phase transformation in each component.

\subsubsection{Unit Cell Model of the Composite}

A well established method to investigate the mechanical response of composites is a parameter study using a suitable mechanical cell model of the composite, which is simulated by means of the finite element method, see for example Mishnaevsky [42].

In this work, this approach has been applied to study the effective stress-strain behavior of this particulate TRIP steel- $\mathrm{ZrO}_{2}$ composite. Details can be found in the publications of Mehlhorn, Prüger et al. [43-45]. The influence of the volume content of $\mathrm{ZrO}_{2}$ particles and the interface properties on the overall response of the composite is investigated. Three different interface types are considered: (i) perfectly bonded, (ii) not bonded, and (iii) cohesive law, respectively, The calculations of the material responses are performed using a finite element analysis of unit cells of the composites under tensile, compressive and biaxial loading. Here, selected results will be reported.

Numerical simulations of composites require proper constitutive equations for both constituents. Here, the Sun model [29] as explained above is employed for the PSZ ceramics. For modeling the viscoplastic deformation and martensitic phase transformation of the TRIP steel, the constitutive law developed by Prüger [46] is applied. It describes the strain-induced transformation from a fully austenitic microstructure $(\gamma)$ to martensite $\left(\alpha^{\prime}\right)$ under thermal and/or mechanical loading. Both material models were available as Fortran routines implemented via UMAT interface into the finite element software ABAQUS [47]. More information about the used material parameters for the PSZ and the specific TRIP-steel can be found in [43-45], and in Chap. 22.

In case of the particular composite, sintered together from steel and ceramic particles, one can assume a representative unit cell consisting of a large number of approximately equally sized and uniformly distributed ceramic particles in a TRIP steel matrix. For simplicity, the embedded ceramic particles are assumed to be spheres. This leads to the unit cell model shown in Fig. 21.21, which is a cube of edge length $2 a$ with a single spherical $\mathrm{ZrO}_{2}$ particle placed in its center.

The mechanical model exhibits a triple symmetry with respect to geometry and loading. Therefore the use of one-eighth of the RVE is admissible, and a corresponding FEM discretization is elaborated. Although the unit cell was numerically simulated under various stress triaxialities, only the results for uniaxial loading are reported here. Regarding the interface between the components, two limiting cases are discussed here: the perfectly bonded connection and the non-bonded, frictionless movable contact. An optimal composite possesses a high energy absorption capacity 


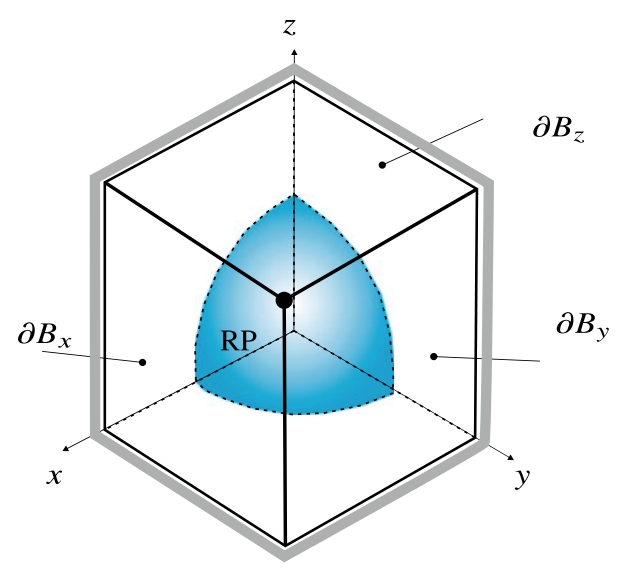

Fig. 21.21 Sketch of unit cell (one-eighth volume) for the TRIP- $\mathrm{ZrO}_{2}$ composite. Symmetry boundary conditions are set on the coordinate planes $x=0, y=0$ and $z=0$. Appropriate displacement and stress boundary conditions are prescribed on opposite planes $\partial B_{x}, \partial B_{y}$ and $\partial B_{z}$ for the different load cases.

The radius $r$ of the particle is adjusted to the volume fraction $f$ of zirconia content by $f(r)=$ $\frac{V_{\text {sphere }}}{V_{\text {cube }}}=\frac{\pi r^{3}}{6 a^{3}}$

and exhibits pronounced phase transformation in the $\mathrm{ZrO}_{2}$ ceramic and the TRIP steel. The macroscopic true stress and true strain tensors $\Sigma$ and $\mathbf{E}$ are used in order to evaluate the mechanical work according to $W=\int_{0}^{\overline{\mathbf{E}}} \Sigma: \mathrm{d} \mathbf{E}$, where $\overline{\mathbf{E}}$ denotes the considered deformation stage. Because the elastic strains are small, $W$ equals approximately the energy absorption for sufficiently large total strains. In order to quantify the relative change in energy absorption capacity, this energy is related to those values $W_{\text {hom }}$ obtained for a unit cell made only of TRIP steel.

During deformation an inhomogeneous distribution of the volume fractions of the monoclinic zirconia and the martensite develop in the ceramic and the TRIP steel, respectively. Therefore the averages of $f_{\alpha^{\prime}}$ and $f_{\mathrm{m}}$ over the corresponding volumes are used. The simulation is stopped, when the maximum principle stress in the PSZ reaches its ultimate tensile strength $\sigma_{\mathrm{cr}}^{\mathrm{t}}=1600 \mathrm{MPa}$.

\subsubsection{Results and Discussion}

The macroscopic true stress and true strain curves are calculated for different variants. The following diagrams show the second invariants $\Sigma^{\mathrm{eq}}$ and $E^{\mathrm{eq}}$ of both variables (to allow comparison with different stress states). The macroscopic strain invariant acts as a loading parameter, whereas the stress and the phase transformation in both components of the composite represent the material response. Table 21.5 summarizes the relative change in energy absorption capability for uniaxial tension. 
Table 21.5 Energy absorption capacity for the composite with perfectly bonded and non-cohesive interface in uniaxial loading

\begin{tabular}{l|l|l|l|l|l|l}
\hline$f$ & 0.05 & 0.05 & 0.10 & 0.10 & 0.20 & 0.20 \\
\hline Interface & Bonded & Non-cohesive & Bonded & Non-cohesive & Bonded & Non-cohesive \\
\hline$W / W_{\text {hom }}$ & 1.06 & 0.92 & 1.16 & 0.85 & 1.37 & 0.72 \\
\hline
\end{tabular}

(a)

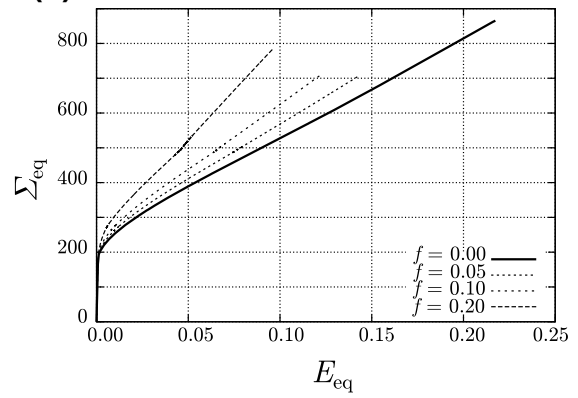

(b)

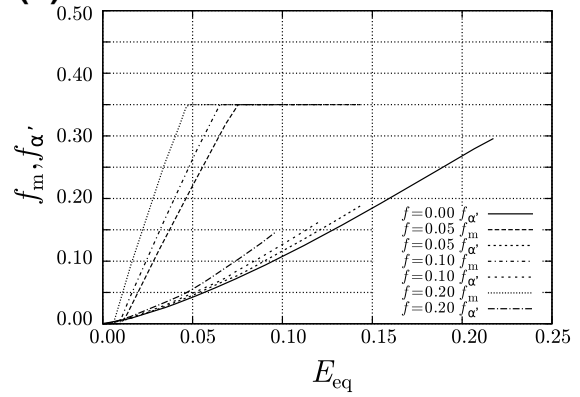

Fig. 21.22 Numerical results for the RVE with perfectly bonded interface in uniaxial loading: a stress-strain diagram and $\mathbf{b}$ phase development curves [43]

In case of the perfectly bonded interface, the stress-strain curves show a distinct dependence on the volume fraction of $\mathrm{ZrO}_{2}$ ceramic $f$, as depicted in Fig. 21.22a. It can be observed that an increasing $f$ leads to higher yield stresses and strain hardening rates compared to the unreinforced TRIP steel $(f=0)$. As consequence, a pronounced increase in the energy absorption $W$ of the composite is obtained. Comparing the values given in Table 21.5, the ratio $W / W^{\text {hom }}$ increases up to more than $35 \%$ (for same macroscopic equivalent strain). Due to the strong interface, load is transferred from the matrix to the reinforcement during deformation of the composite. Therefore high stresses occur in the ceramic, which reduces the maximal attainable strain with increasing zirconia content $f$. Regarding the phase transformation behavior, a higher volume fraction of zirconia $f$ enhances the tendency to phase transformation in zirconia as well as in TRIP steel (Fig. 21.22b). The phase transformation capacity in the PSZ component is saturated to the maximum of 35\% in a smaller strain interval. The tendency to phase transformation in the TRIP steel $f_{\alpha}^{\prime}$ increases at higher zirconia content, but is limited due to failure of the ceramic.

In case of a non-cohesive interface, both the initial yield stress and the strain hardening rate tend to decrease with higher zirconia content $f$, see Fig. 21.23a. Figure $21.23 \mathrm{~b}$ shows that the development of martensite is considerably higher than in the case of the perfectly bonded interface. At the end of deformation nearly $30 \%$ of martensite has evolved. Because of the non-cohesive interface, no tensile stresses are transferred from the TRIP steel matrix to the zirconia inclusion. Thus, no phase transformation is seen in zirconia. Moreover, the area of the load bearing crosssection consists of TRIP steel only and is the smaller the higher the zirconia content 
(a)

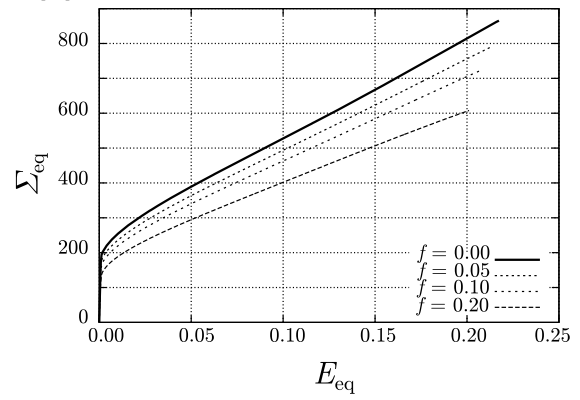

(b)

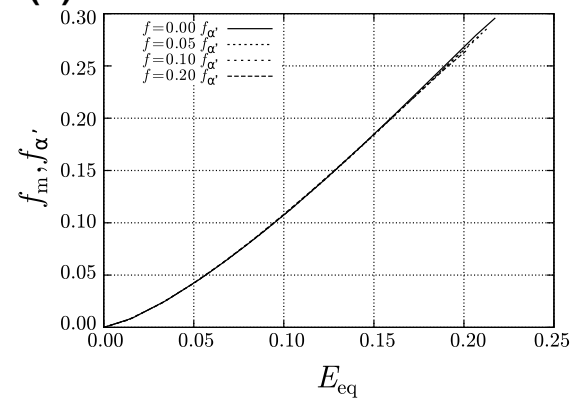

Fig. 21.23 Numerical results for the RVE with non-cohesive interface in uniaxial loading: a stressstrain diagram and $\mathbf{b}$ phase development curves [43]

becomes, which reduces macroscopic yield stress. After debonding, the particle acts partially like a void. However, no softening is observed in the macroscopic stressstrain response because of the hardening behavior of the TRIP steel and the locking effect caused by the particle.

One can conclude that the behavior of the real composite material lies between the two extreme cases considered here, since the interface between ceramic and steel has a finite strength. Moreover, the impact of particle reinforcement is stronger under compressive loading [45].

\subsection{Conclusions}

Based on the work of Levitas and Preston [13] for generic martensitic transformation, a phase-field model for $\mathrm{MgO}-\mathrm{ZrO}_{2}$ material was implemented [16]. The potential function used in this work results in a proper representation of transformation behavior of zirconia ceramics from a pure thermodynamic stand point. In the simulations different patterns of microstructures were found for cooling induced and stress induced transformation. These patterns are consistent with experimental observations by Hannink et al. [18]. It is evident that the presence of an energy barrier plays a key role in variant selection and the transformation path taken. By which, in stress induced case a sequential growth of lamellae was visible. In contrast, the cooling induced case is categorized with an almost homogeneous nucleation where all variants are preferred to evolve. Additionally on a single crystal level the simulations showed that, in the stress induced case, microstructure with junction planes parallel to the ' $c$ '-axis is formed because of variant selection. It was shown that residual stresses inside $t$-phase lentils from $c \rightarrow t$ transformation have a magnitude of $\approx 0.3 \mathrm{GPa}$ and contribute to the stability of the $t$-phase. Also the magnitude of stress required for introducing energy barrier increases with decreasing temperature below $M_{s}$. 
A mesomechanical, two-dimensional model for Mg-PSZ with an energetic transformation criterion based on the analytical solution for a rectangular inclusion in an infinite matrix has been successfully developed. Using this model the influence of individual parameters such as size, geometry and surface energies on the transformation initiation and resulting microstructure can be efficiently studied. It predicts that the stability of the tetragonal inclusions deteriorates as the inclusions grow in size and aspect-ratio. Further, the tension-compression asymmetry of the transformation behavior known from experiments is captured correctly. A homogenization approach based on the Mori-Tanaka method predicts the transformation to be auto-catalytic within a grain.

A continuum material model for transformation plasticity in partially stabilized zirconia ceramics has been further developed to account for (i) particle size dependent phase transformation behavior, (ii) temperature dependent phase transformation, and (iii) thermoelastic deformation. These more physically based features lead to a nonlinear hardening behavior and smoothly rounded hysteresis curves for the strain and the generated monoclinic phase fraction during a temperature cycle. The influence of the tetragonal particle size distribution on phase transformation could be predicted qualitatively quite well.

Finally, the mechanical properties of a TRIP steel matrix reinforced by $\mathrm{ZrO}_{2}$ particles are analyzed, taking the phase transformation in both constituents into account. The influence of the volume content and the interface properties of $\mathrm{ZrO}_{2}$ particles on the overall response of the composite is investigated. Material variants with three different zirconia contents and two different interface types, perfectly bonded and non-cohesive, respectively, are considered. The calculations of the material responses are performed using a finite element analysis of representative volume elements of the composites under tensile, compressive and biaxial loading. The results indicate that the enrichment of the TRIP steel with zirconia particles leads to a significant strengthening effect provided the interface has cohesive properties.

Acknowledgements The authors gratefully acknowledge the the German Research Foundation or Deutsche Forschungsgemeinschaft (DFG) for supporting this work in subproject C4, and was created as part of the Collaborative Research Center TRIP-Matrix-Composites (Project number 54473466-CRC 799). We appreciate the contributions of former colleagues Dr.-Ing. Uwe Mühlich, Dr.-Ing. Stefan Prüger, and Lars Mehlhorn to the achievements.

\section{References}

1. S. Decker, L. Krüger, S. Richter, S. Martin, U. Martin, Steel Res. Int. 83(6), 521 (2012)

2. R.C. Garvie, R.H.J. Hannink, R.T. Pascoe, Nature 258(5537), 703 (1975)

3. A.G. Evans, A.H. Heuer, J. Am. Ceram. Soc. 63(5-6), 241 (1980)

4. A.G. Evans, N. Burlingame, M. Drory, W.M. Kriven, Acta Metall. 29(2), 447 (1981)

5. F.F. Lange, J. Mater. Sci. 17(1), 240 (1982)

6. T. Hensl, U. Mühlich, M. Budnitzki, M. Kuna, Acta Mater. 86, 361 (2014)

7. R.C. Garvie, J. Phys. Chem. 69(4), 1238 (1965)

8. Y. Wang, H. Wang, L.Q.Q. Chen, A.G. Khachaturyan, J. Am. Ceram. Soc. 76(12), 3029 (1993) 
9. Y. Wang, H.Y.Y. Wang, L.Q.Q. Chen, A.G. Khachaturyan, J. Am. Ceram. Soc. 78(3), 657 (1995)

10. Y. Wang, A.G. Khachaturyan, Acta Mater. 45(2), 759 (1997)

11. M. Mamivand, M.A. Zaeem, H.E. Kadiri, L.Q. Chen, Acta Mater. 61(14), 5223 (2013)

12. M. Mamivand, M. Asle Zaeem, H. El Kadiri, Int. J. Plast. 60, 71 (2014)

13. V. Levitas, D. Preston, Three-dimensional Landau theory for multivariant stress-induced martensitic phase transformations I Austenite $<->$ martensite. Phys. Rev. B 66(13), 1-9 (2002). https://doi.org/10.1103/PhysRevB.66.134206

14. V. Levitas, D. Preston, Three-dimensional Landau theory for multivariant stress-induced martensitic phase transformations. II. Multivariant phase transformations and stress space analysis. Phy. Rev. B 66(13), 1-15. https://doi.org/10.1103/PhysRevB.66.134207

15. V. Levitas, D.L. Preston, D.W. Lee, Phys. Rev. B 68(13), 1 (2003)

16. M.K. Rajendran, M. Kuna, M. Budnitzki, Undercooling versus stress induced martensitic phase transformation: the case of $\mathrm{MgO}$ - partially stabilized zirconia. Comput. Mater. Sci. 174, 109460 (2019). https://doi.org/10.1016/j.commatsci.2019.109460

17. P.M. Kelly, C.J. Ball, J. Am. Ceram. Soc. 69(3), 259 (1986)

18. R.H.J. Hannink, P.M. Kelly, B.C. Muddle, J. Am. Ceram. Soc. 83(3), 461 (2000)

19. X.S. Zhao, S.L. Shang, Z.K. Liu, J.Y. Shen, J. Nucl. Mater. 415(1), 13 (2011)

20. H. Lukas, S. Fries, B. Sundman, Computational Thermodynamics-The CALPHAD Method (Cambridge University Press, 2007)

21. D. Pavlyuchkov, G. Savinykh, O. Fabrichnaya, J. Eur. Ceram. Soc. 34(5), 1397 (2014)

22. L.Q. Chen, W. Yang, Phys. Rev. B 50(21), 15752 (1994)

23. G.K. Bansal, A.H. Heuer, Acta Metall. 20(11), 1281 (1972)

24. G.K. Bansal, A.H. Heuer, Acta Metall. 22(4), 409 (1974)

25. R.C. Garvie, J. Phys. Chem. 82(2), 218 (1978)

26. C.R. Chen, S.X. Li, Q. Zhang, Mater. Sci. Eng. A 272(2), 398 (1999)

27. W.E. Lee, M. Rainforth, Ceramic Microstructures Property Control by Processing (Chapman \& Hall, 1994)

28. R. Garvie, M. Swain, J. Mater. Sci. 20, 1193 (1985)

29. Q.P. Sun, K.C. Hwang, S.W. Yu, J. Mech. Phys. Solids 39(4), 507 (1991)

30. C. Wang, M. Zinkevich, F. Aldinger, J. Am. Ceram. Soc. 89(12), 3751 (2006)

31. I.W. Chen, Y.H. Chiao, Acta Metall. 31(10), 1627 (1983)

32. T. Mori, K. Tanaka, Acta Metall. 21(5), 571 (1973)

33. Y. Benvensite, Mech. Mater. 6(2), 147 (1987)

34. F.D. Fischer, Q.P. Sun, K. Tanaka, Appl. Mech. Rev. 49(6), 317 (1996)

35. J.R. Rice, J. Mech. Phys. Solids 19(6), 433 (1971)

36. Abaqus, Abaqus, Online documentation, 6th edn. (Dassault Systems, 2014)

37. L. Mehlhorn, U. Mühlich, M. Kuna, Adv. Eng. Mater. 15(7), 638 (2013)

38. D. Gross, T. Seelig, Bruchmechanik-Mit einer Einführung in die Mikromechanik, 4th edn. (Springer, Berlin Heidelberg, 2007)

39. A. Puzrin, G. Houlsby, Int. J. Plast. 16(9), 1017 (2000)

40. T. Mura, Mechanics of elastic and inelastic solids, in Micromechanics of Defects in Solids (Springer, 1991)

41. D.B. Marshall, J. Am. Ceram. Soc. 69(3), 173 (1986)

42. L.L. Mishnaevsky, Acta Mater. 52(14), 4177 (2004)

43. L. Mehlhorn, S. Prüger, S. Soltysiak, U. Mühlich, M. Kuna, Steel Res. Int. 82(9), 1022 (2011)

44. S. Prüger, L. Mehlhorn, S. Soltysiak, M. Kuna, Comput. Mater. Sci. 64, 273 (2012)

45. S. Prüger, L. Mehlhorn, U. Mühlich, M. Kuna, Adv. Eng. Mater. 15(7), 542 (2013)

46. S. Prüger, Thermomechanische Modellierung der dehnungsinduzierten Phasenumwandlung und der asymmetrischen Verfestigung in einem TRIP-Stahlguss. Ph.D. thesis, TU Bergakademie Freiberg, 2016

47. Hibbitt, Karlsson, Sorenson, ABAQUS: Version 6.7 Documentation (HKS, 2009) 
Open Access This chapter is licensed under the terms of the Creative Commons Attribution 4.0 International License (http://creativecommons.org/licenses/by/4.0/), which permits use, sharing, adaptation, distribution and reproduction in any medium or format, as long as you give appropriate credit to the original author(s) and the source, provide a link to the Creative Commons license and indicate if changes were made.

The images or other third party material in this chapter are included in the chapter's Creative Commons license, unless indicated otherwise in a credit line to the material. If material is not included in the chapter's Creative Commons license and your intended use is not permitted by statutory regulation or exceeds the permitted use, you will need to obtain permission directly from the copyright holder. 